\title{
The Mrk 231 molecular outflow as seen in $\mathrm{OH}^{\star}$
}

\author{
E. González-Alfonso ${ }^{1}$, J. Fischer ${ }^{2}$, J. Graciá-Carpio ${ }^{3}$, N. Falstad ${ }^{4}$, E. Sturm ${ }^{3}$, M. Meléndez ${ }^{5}$, H. W. W. Spoon ${ }^{6}$, \\ A. Verma ${ }^{7}$, R. I. Davies 3 , D. Lutz ${ }^{3}$, S. Aalto ${ }^{4}$, E. Polisensky ${ }^{2}$, A. Poglitsch ${ }^{3}$, S. Veilleux ${ }^{5}$, and A. Contursi ${ }^{3}$ \\ ${ }^{1}$ Universidad de Alcalá, Departamento de Física y Matemáticas, Campus Universitario, 28871 Alcalá de Henares, Madrid, Spain \\ e-mail: eduardo.gonzalez@uah.es \\ 2 Naval Research Laboratory, Remote Sensing Division, 4555 Overlook Ave SW, Washington DC 20375, USA \\ 3 Max-Planck-Institute for Extraterrestrial Physics (MPE), Giessenbachstraße 1, 85748 Garching, Germany \\ ${ }^{4}$ Department of Earth and Space Sciences, Chalmers University of Technology, Onsala Space Observatory, Onsala, Sweden \\ 5 Department of Astronomy, University of Maryland, College Park, MD 20742, USA \\ ${ }^{6}$ Cornell University, Astronomy Department, Ithaca NY 14853, USA \\ 7 University of Oxford, Oxford Astrophysics, Denys Wilkinson Building, Keble Road, Oxford OX1 3RH, UK
}

Received 16 April 2013 / Accepted 2 October 2013

\section{ABSTRACT}

\begin{abstract}
We report on the Herschel/PACS observations of $\mathrm{OH}$ in Mrk 231, with detections in nine doublets observed within the PACS range, and present radiative-transfer models for the outflowing $\mathrm{OH}$. Clear signatures of outflowing gas are found in up to six $\mathrm{OH}$ doublets with different excitation requirements. At least two outflowing components are identified, one with $\mathrm{OH}$ radiatively excited, and the other with low excitation, presumably spatially extended and roughly spherical. Particularly prominent, the blue wing of the absorption detected in the in-ladder ${ }^{2} \Pi_{3 / 2} J=9 / 2-7 / 2 \mathrm{OH}$ doublet at $65 \mu \mathrm{m}$, with $E_{\text {lower }}=290 \mathrm{~K}$, indicates that the excited outflowing gas is generated in a compact and warm (circum)nuclear region. Because the excited, outflowing $\mathrm{OH}$ gas in Mrk 231 is associated with the warm, far-infrared continuum source, it is most likely more compact (diameter of $\sim 200-300 \mathrm{pc}$ ) than that probed by CO and $\mathrm{HCN}$. Nevertheless, its mass-outflow rate per unit of solid angle as inferred from $\mathrm{OH}$ is similar to that previously derived from $\mathrm{CO}, \gtrsim 70 \times\left(2.5 \times 10^{-6} / X_{\mathrm{OH}}\right) M_{\odot} \mathrm{yr}^{-1} \mathrm{sr}^{-1}$, where $X_{\mathrm{OH}}$ is the $\mathrm{OH}$ abundance relative to $\mathrm{H}$ nuclei. In spherical symmetry, this would correspond to $\gtrsim 850 \times\left(2.5 \times 10^{-6} / X_{\mathrm{OH}}\right) M_{\odot} \mathrm{yr}^{-1}$, though significant collimation is inferred from the line profiles. The momentum flux of the excited component attains $\sim 15 L_{\mathrm{AGN}} / c$, with an $\mathrm{OH}$ column density of $(1.5-3) \times 10^{17} \mathrm{~cm}^{-2}$ and a mechanical luminosity of $\sim 10^{11} L_{\odot}$. In addition, the detection of very excited, radiatively pumped $\mathrm{OH}$ peaking at central velocities indicates the presence of a nuclear reservoir of gas rich in $\mathrm{OH}$, plausibly the $130 \mathrm{pc}$ scale circumnuclear torus previously detected in $\mathrm{OH}$ megamaser emission, that may be feeding the outflow. An exceptional ${ }^{18} \mathrm{OH}$ enhancement, with $\mathrm{OH} /{ }^{18} \mathrm{OH} \lesssim 30$ at both central and blueshifted velocities, is most likely the result of interstellar-medium processing by recent starburst and supernova activity within the circumnuclear torus or thick disk
\end{abstract}

Key words. line: formation - galaxies: ISM - ISM: jets and outflows - infrared: galaxies - galaxies: individual: Mrk 231

\section{Introduction}

Current models of galaxy evolution involve galactic-scale outflows driven by starbursts and active galactic nuclei (AGN) as key ingredients. The outflows trace the negative feedback from AGN and/or star formation on the molecular gas, eventually shutting off the feeding process and quenching the growth of the stellar population and/or of the supermassive black hole (e.g. di Matteo et al. 2005). AGN feedback could be responsible for the observed black hole mass-velocity dispersion relationship (Murray et al. 2005) and create a population of red gas-poor ellipticals. In the past, outflows have been observed in many starbursts and quasi-stellar objects (QSOs), mostly in the ionized and neutral atomic gas component (e.g. Veilleux et al. 2005, for a review).

Molecular gas may dominate the mass-outflow rate of outflows, providing important constraints on the timescale for dispersing the (circum)nuclear gas in the host galaxy. Molecular outflows have been reported in several galaxies at millimeter wavelengths (e.g. Baan et al. 1989; Walter et al. 2002; Sakamoto et al. 2009). The discovery of a massive molecular outflow in

\footnotetext{
* Herschel is an ESA space observatory with science instruments provided by European-led Principal Investigator consortia and with important participation from NASA.
}

Mrk 231, an ultraluminous infrared galaxy (ULIRG) harboring the closest quasar known, in Herschel/PACS (Pilbratt et al. 2010; Poglitsch et al. 2010) spectroscopy is a key finding. The outflows are traced by P-Cygni OH line profiles in the 79 and $119 \mu \mathrm{m}$ doublets, and in the high-lying $65 \mu \mathrm{m}$ doublet $\left(E_{\text {lower }} \approx 300 \mathrm{~K}\right.$ ), with high-velocity shifts of $>1000 \mathrm{~km} \mathrm{~s}^{-1}$ (Fischer et al. 2010; Sturm et al. 2011, hereafter F10 and S11, respectively). Analysis and model fits of these lines yielded a preliminary mass-outflow rate of $\dot{M} \sim 10^{3} M_{\odot} \mathrm{y}^{-1}$. The extreme outflow was also detected at millimeter wavelengths in $\mathrm{CO}$, giving a similar $\dot{M}$ (Feruglio et al. 2010), and in $\mathrm{HCN}, \mathrm{HCO}^{+}$, and $\mathrm{HNC}$ (Aalto et al. 2012). Recently, Cicone et al. (2012) have found that the outflowing $\mathrm{CO}$ is not highly excited relative to the quiescent gas, and that the outflow size decreases with increasing critical density of the transition. From neutral Na I D absorption, Rupke \& Veilleux (2011) estimated a similar $\dot{M} \sim 400 M_{\odot} \mathrm{y}^{-1}$ on spatial scales of $\sim 3 \mathrm{kpc}$.

Relative to $\mathrm{CO}$, the specific characteristic of $\mathrm{OH}$ in galaxies is that the high-lying lines are radiatively (instead of collisionally) excited, and thus selectively trace an outflow region close to the circumnuclear source of strong far-IR radiation density (González-Alfonso et al. 2008). In addition to tracing the gas, the lines also probe the coexisting warm dust responsible for the observed excitation. Although the outflow is not spatially resolved, 
Table 1. Herschel/PACS observations of $\mathrm{OH}$ in Mrk 231.

\begin{tabular}{|c|c|c|c|c|c|}
\hline Transition & $\begin{array}{c}\text { Rest wavelengths }{ }^{a} \\
(\mu \mathrm{m})\end{array}$ & Program $^{b}$ & Obs. $\mathrm{ID}^{c}$ & $\begin{array}{c}\text { Number of } \\
\text { spatial pixels }^{d}\end{array}$ & $\begin{array}{c}\sigma \\
(\mathrm{Jy})\end{array}$ \\
\hline $\mathrm{OH} \Pi_{3 / 2}-\Pi_{3 / 2} \frac{5}{2}-\frac{3}{2}$ & $119.233-119.441$ & GT & 1342186811 & 1 & 0.29 \\
\hline \multirow{3}{*}{$\mathrm{OH} \Pi_{1 / 2}-\Pi_{3 / 2} \frac{1}{2}-\frac{2}{2}$} & $79.118-79.181$ & GT & 1342186811 & 1 & 0.26 \\
\hline & & GT & 1342186811 & 1 & 0.26 \\
\hline & & OT2 & 1342253536 & 1 & 0.23 \\
\hline $\mathrm{OH} \Pi_{1 / 2}-\Pi_{3 / 2} \frac{3}{2}-\frac{3}{2}$ & $53.261-53.351$ & OT2 & 1342253530 & 1 & 0.24 \\
\hline $\mathrm{OH} \Pi_{3 / 2}-\Pi_{3 / 2} \frac{7}{2}-\frac{5}{2}$ & $84.420-84.597$ & OT2 & 1342253537 & 3 & 0.50 \\
\hline \multirow{3}{*}{$\mathrm{OH} \Pi_{3 / 2}-\Pi_{3 / 2} \frac{9}{2}-\frac{7}{2}$} & $65.132-65.279$ & GT & 1342207782 & 1 & 0.19 \\
\hline & & GT & 1342207782 & 3 & 0.24 \\
\hline & & OT2 & 1342253532 & 3 & 0.25 \\
\hline $\mathrm{OH} \Pi_{1 / 2}-\Pi_{1 / 2} \frac{7}{2}-\frac{5}{2}$ & $71.171-71.216$ & OT2 & 1342253534 & 3 & 0.36 \\
\hline $\mathrm{OH} \Pi_{3 / 2}-\Pi_{3 / 2} \frac{11}{2}-\frac{9}{2}$ & $52.934-53.057$ & OT2 & 1342253530 & 1 & 0.24 \\
\hline $\mathrm{OH} \Pi_{1 / 2}-\Pi_{1 / 2} \frac{9}{2}-\frac{7^{2}}{2}$ & $55.891-55.950$ & OT2 & 1342253531 & 3 & 0.19 \\
\hline $\mathrm{OH} \Pi_{1 / 2}-\Pi_{1 / 2} \frac{3}{2}-\frac{1}{2}$ & $163.124-163.397$ & OT1 & 1342223369 & 1 & 0.25 \\
\hline
\end{tabular}

Notes. ${ }^{(a)}$ The two values correspond to the two $\Lambda$-components of the doublets; each one is the average of the two hyperfine transitions that make up a component. ${ }^{(b)}$ GT and OT indicate "guaranteed time" and "open time". ${ }^{(c)}$ Identification number. ${ }^{(d)}$ Number of spatial pixels used to generate the spectrum. In case of 1, the central pixel was used; in case of 3, the spectra from the three brightest spatial pixels were coadded. In all cases, the absolute fluxes were scaled to match the total flux as obtained by coadding the fluxes from the 25 pixels.

the observed excitation conditions provide information about the spatial extent of the outflow, which enables the estimation of the outflow physical parameters (mass-outflow rate, mechanical power and energy). The high-velocity molecular outflows were found to be common in local ULIRGs, and preliminary evidence suggested that higher AGN luminosities (and higher AGN contributions to $L_{\mathrm{IR}}$ ), as determined by mid-IR diagnostics, correlate with higher terminal velocities and shorter gas depletion timescales (S11). This result has been recently confirmed by Spoon et al. (2013) and Veilleux et al. (2013) based on larger sample sizes.

In this work, we present the velocity profiles and fluxes of all of the $\mathrm{OH}$ and ${ }^{18} \mathrm{OH}$ doublets seen in the Herschel/PACS spectroscopic observations of Mrk 231, and an analysis of these profiles and fluxes based on radiative-transfer modeling. In Sect. 2 we discuss the details of the observations and give an overview of the general characteristics of the profiles, together with qualitative assessments on the excitation conditions, optical depths, far-IR extinction, geometry, and ${ }^{16} \mathrm{O} /{ }^{18} \mathrm{O}$ abundance ratio in the circumnuclear region of Mrk 231. In Sect. 3, we discuss the radiative-transfer models that are used to quantitatively analyze the observations, and the motivation for, properties of, and derived parameters of the several components that we use to characterize the gas seen in $\mathrm{OH}$. In Sect. 4, we summarize the picture that emerges from the observations and the modeled components, their relationship to structures seen in other diagnostics, and the implications for the role of the AGN and circumnuclear starburst. We adopt a distance to Mrk 231 of $192 \mathrm{Mpc}$ $\left(H_{0}=70 \mathrm{~km} \mathrm{~s}^{-1} \mathrm{Mpc}^{-1}, \Omega_{\Lambda}=0.73\right.$, and $\left.z=0.04218\right)$.

\section{Observations and description of the spectra}

Following the detection of outflows traced by the ground-state $\mathrm{OH}$ doublets at 119 and $79 \mu \mathrm{m}$ and of the excited OH $65 \mu \mathrm{m}$ transition, based on the guaranteed-time key program SHINING observations (hereafter GT observations, PI: E. Sturm; F10; S11), completion of the full (52.3-98, 104.6-196 $\mu \mathrm{m})$, highresolution PACS spectrum of Mrk 231 was carried out on October 16 (2012) as part of the open time-2 Herschel phase (hereafter OT2 observations; PI: J. Fischer). The spectrum of the
$\mathrm{OH} \Pi_{1 / 2} J=3 / 2-1 / 2$ doublet at $163 \mu \mathrm{m}$ was taken from an OT1 program (PI: R. Meijerink). The GT observations and reduction process were described in F10 and S11. For the OT2 and OT1 observations, the spectra were also taken in high spectral sampling density mode using first and second orders of the grating. The velocity resolution of PACS in first order ranges from $\approx 320 \mathrm{~km} \mathrm{~s}^{-1}$ at $105 \mu \mathrm{m}$ to $\approx 180 \mathrm{~km} \mathrm{~s}^{-1}$ at $190 \mu \mathrm{m}$, and in second order from $\approx 210 \mathrm{~km} \mathrm{~s}^{-1}$ at $52 \mu \mathrm{m}$ to $\approx 110 \mathrm{~km} \mathrm{~s}^{-1}$ at 98 microns. The data reduction was carried out mostly using the standard PACS reduction and calibration pipeline (ipipe) included in HIPE 6.0 and HIPE 10.0. The two HIPE versions yielded essentially identical continuum-normalized spectra, with the continuum level from v10 stronger than that from v6 by up to $\sim 10 \%$.

We focus in this work on the observations of the $\mathrm{OH}$ doublets, which are summarized in Table 1 and Fig. 1 and are displayed in Fig. 2. Spectroscopic parameters used for line identification and radiative-transfer modeling were taken from the spectral-line catalogs of the CDMS (Müller et al. 2001, 2005) and JPL (Pickett et al. 1998). All nine OH doublets within the PACS wavelength range, having lower-level energies up to $E_{\text {lower }}=620 \mathrm{~K}$, were detected and are indicated in the energy-level diagram of Fig. 1. These are the same transitions as were detected in NGC 4418 and Arp 220 (González-Alfonso et al. 2012, hereafter G-A12), except for the cross-ladder $\Pi_{1 / 2}-\Pi_{3 / 2} J=3 / 2-5 / 2$ line at $\lambda_{\text {rest }}=96 \mu \mathrm{m}(\mathrm{de}-$ tected in NGC 4418) that is redshifted in Mrk 231 into the gap at $\approx 100 \mu \mathrm{m}$ between the green and red bands. For simplicity, we denote a given doublet by using its rounded wavelength as indicated in Fig. 1 (e.g. OH119). The ${ }^{18} \mathrm{OH}$ doublets, lying close in wavelength to the $\mathrm{OH}$ transitions, were also observed and unambiguously detected at 120,85 , and $65 \mu \mathrm{m}$. Spectroscopic parameters for the ${ }^{17} \mathrm{OH}$ doublets were taken from Polehampton et al. (2003); the positions of the ${ }^{17} \mathrm{OH} 119$ and ${ }^{17} \mathrm{OH} 84$ doublets, expected to have the strongest signatures, are indicated in Figs. 2a and $\mathrm{c}$. There is no evidence in the spectra for either absorption or emission attributable to ${ }^{17} \mathrm{OH}$, whose transitions fall between those of the two more abundant isotopologs.

The abscissas in Fig. 2 indicate the velocity relative to the shorter-wavelength component (hereafter, blue component) of 


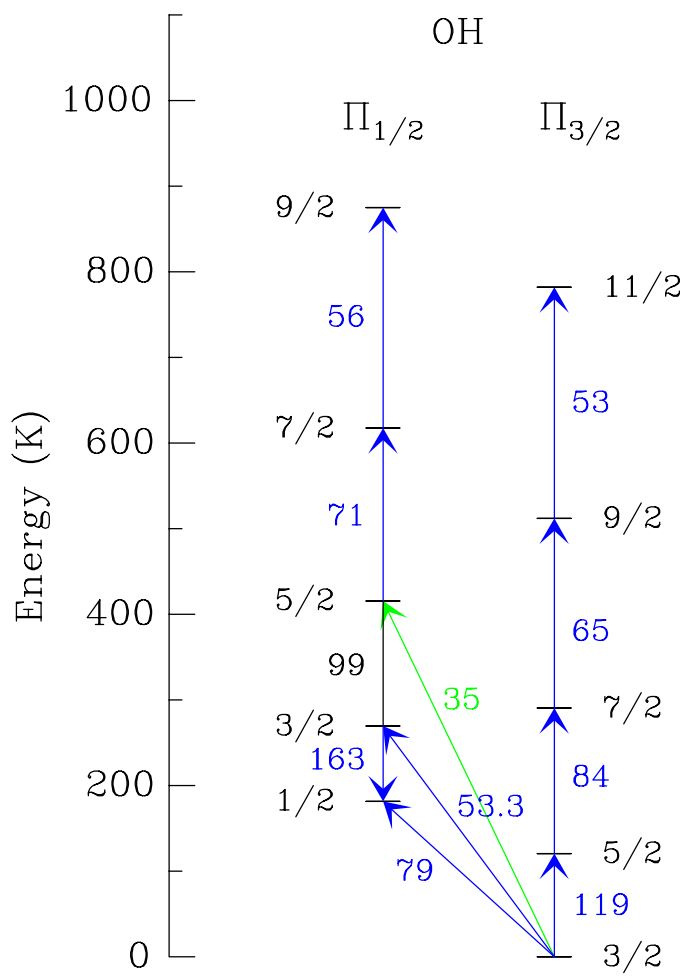

Fig. 1. Energy level diagram of $\mathrm{OH}$ showing the transitions detected in Mrk 231 with Herschel/PACS and Spizer/IRS with blue and green arrows, respectively. The $l$-doubling splitting of the levels is not indicated. Colored numbers indicate rounded wavelengths in $\mu \mathrm{m}$. The $\Pi_{1 / 2} J=5 / 2-3 / 2$ (black line) and the $\Pi_{1 / 2}-\Pi_{3 / 2} J=3 / 2-5 / 2$ doublets at $\lambda_{\text {rest }} \approx 99$ and $96 \mu \mathrm{m}$ (the latter detected in NGC 4418, G-A12) were not observed, as they are redshifted into the PACS gap at $100 \mu \mathrm{m}$. In the text, we denote a doublet by giving its wavelength (e.g. OH119).

each doublet, and are calculated for a redshift of $z=0.04218$. The positions of the $\mathrm{OH}$ and ${ }^{18} \mathrm{OH}$ doublets are indicated with black arrows, while those of potentially contaminating lines of other species (discussed in Sect. 2.2) are indicated with blue arrows. In panel $b$, three independent spectra of the OH79 doublet are shown for comparison; they are listed in Table 1.

To characterize the molecular outflow traced by the $\mathrm{OH}$ lines, it is important to have a flat baseline that minimizes the uncertainties in the continuum level, and thus in the velocity extent of the line wings. The central spatial pixel (spaxel) of the $5 \times 5$ spaxels of PACS gives the highest signal-to-noise ratio $(\mathrm{S} / \mathrm{N})$ spectrum, and was adopted whenever the continuum level was flat and the baseline was well characterized. However, the continuum level from the central spaxel shows low-level fluctuations at some wavelengths that probably result from small pointing drift motions. In these cases, we coadded the flux densities resulting from the three brightest spaxels, which resulted in flat baselines at the expense of a lower S/N. Regardless of the number of spaxels used, the resulting spectra were re-scaled to the continuum level of all 25 spaxels combined (to account for point spread function losses and pointing uncertainties, S11). Table 1 lists the number of spaxels used to generate the spectrum of each $\mathrm{OH}$ doublet, and the baselines are indicated in Fig. 2 with dashed lines.

We also analyzed the $\mathrm{OH} 35$ ground-state doublet observed in the Spitzer/IRS long-high spectrum of Mrk 231. The spectrum, presented in Fig. 3, is the result of combining eight independent observations of the source obtained between 2006 and 2009 as part of the IRS calibration program (earlier versions of the spectrum can be found in Farrah et al. 2007 and Armus et al. 2007).

\subsection{General characteristics of the $\mathrm{OH}$ spectra}

The $\mathrm{OH}$ spectra displayed in Fig. 2 show a diversity of line shapes. The ground-state $\mathrm{OH} 119$ and $\mathrm{OH} 79$ doublets (panels a and $\mathrm{b}$ ) exhibit prominent P-Cygni profiles, indicative of outflowing gas with the absorption produced in front of, and the emission feature laterally adjacent to and behind the far-IR source. Absorption in $\mathrm{OH} 119$ is found up to a blueshifted velocity of $-1600 \mathrm{~km} \mathrm{~s}^{-1}$, while the case of $\mathrm{OH} 79$ is uncertain due to contamination by $\mathrm{H}_{2} \mathrm{O}$. Emission in $\mathrm{OH} 79$ is detected up to a velocity of $\approx 770 \mathrm{~km} \mathrm{~s}^{-1}$ from the red $\mathrm{OH}$ component. The third ground-state transition within the PACS range, the OH53.3 doublet (panel e), does not show any emission feature. Its blueshifted absorption extends up to at least $-1000 \mathrm{~km} \mathrm{~s}^{-1}$, being contaminated by the very high-lying OH53 doublet at more negative velocities (also shown in panel e). The Spitzer/IRS OH35 spectrum (Fig. 3), which has significantly lower spectral resolution $\left(500 \mathrm{~km} \mathrm{~s}^{-1}\right)$, shows absorption that peaks at central velocities with no emission feature; the absorption on the blue side is more prominent than on the red side. Detection of OH79, $\mathrm{OH} 53.3$, and $\mathrm{OH} 35$ implies that $\mathrm{OH} 119$, with a much higher opacity (F10), is optically thick. However, the peak absorption in $\mathrm{OH} 119$ is only $30 \%$ of the continuum, indicating that the $\mathrm{OH} 119$ doublet at a given velocity only covers a fraction of the total $119 \mu \mathrm{m}$ continuum, and/or that the $119 \mu \mathrm{m}$ transition is very excited ${ }^{1}$.

The excited OH84 and OH65 doublets (panels c and d) do not show any emission feature either, but display prominent blueshifted absorption. It is worth noting that while OH65 shows absorption up to $\sim-1500 \mathrm{~km} \mathrm{~s}^{-1}$, the less excited OH84 doublet (with lower $\mathrm{S} / \mathrm{N}$ ) only shows absorption up to $\sim-1000 \mathrm{~km} \mathrm{~s}^{-1}$. The reliability of the extreme OH65 blueshifted absorption is discussed in Sect. 2.3.

While the peak absorption in the OH119 and OH79 doublets is blueshifted by -300 and $-240 \mathrm{~km} \mathrm{~s}^{-1}$, respectively, relative to the blue component of the doublet, the OH53.3 and OH35 peak closer to central velocities. The increase in line excitation along the $\Pi_{3 / 2}$ ladder also shifts the peak absorption toward rest velocities, with the high-lying $\mathrm{OH} 65$ and $\mathrm{OH} 53$ doublets peaking at nearly $v=0 \mathrm{~km} \mathrm{~s}^{-1}$. The latter transition does not show any hint of blueshifted absorption to within the $\mathrm{S} / \mathrm{N}$. These velocity shifts suggest that the excited lines trace an outflow region not entirely coincident with that probed by the ground-state OH119 and OH79. The OH119 and OH65 spectra also show blueshifted absorption at velocities significantly higher than the line wing emission in $\mathrm{CO}$ and $\mathrm{HCN}$, which is observed just out to $\sim 800 \mathrm{~km} \mathrm{~s}^{-1}$ (Feruglio et al. 2010; Cicone et al. 2012; Aalto et al. 2012).

Along the $\Pi_{1 / 2}$ ladder, the high-lying $\mathrm{OH} 71$ doublet (panel f) also peaks at rest velocities, with possible blueshifted absorption that is uncertain due to the proximity of a strong $\mathrm{H}_{2} \mathrm{O}$ line at $\approx-500 \mathrm{~km} \mathrm{~s}^{-1}$. Hints of emission are also seen at redshifted velocities in the $\mathrm{OH} 71$ doublet.

The only $\mathrm{OH}$ transition with emission dominating over absorption is the $\mathrm{OH} 163$ doublet (panel h). The features are slightly redshifted (by $\approx 50 \mathrm{~km} \mathrm{~s}^{-1}$ ), and there are hints of redshifted emission at velocities higher than $700 \mathrm{~km} \mathrm{~s}^{-1}$ (in line with the emission feature in $\mathrm{OH} 79$ at extreme, positive velocities). The OH163 doublet also shows blueshifted absorption up to $-350 \mathrm{~km} \mathrm{~s}^{-1}$, as well as a relatively weak emission feature at $-480 \mathrm{~km} \mathrm{~s}^{-1}$ that is probably attributable to $\left(\sim 100 \mathrm{~km} \mathrm{~s}^{-1}\right.$ redshifted) $\mathrm{CO}(16-15)$.

1 The absorption strength of the transition approaches zero if the excitation temperature of the transition approaches the dust temperature. 

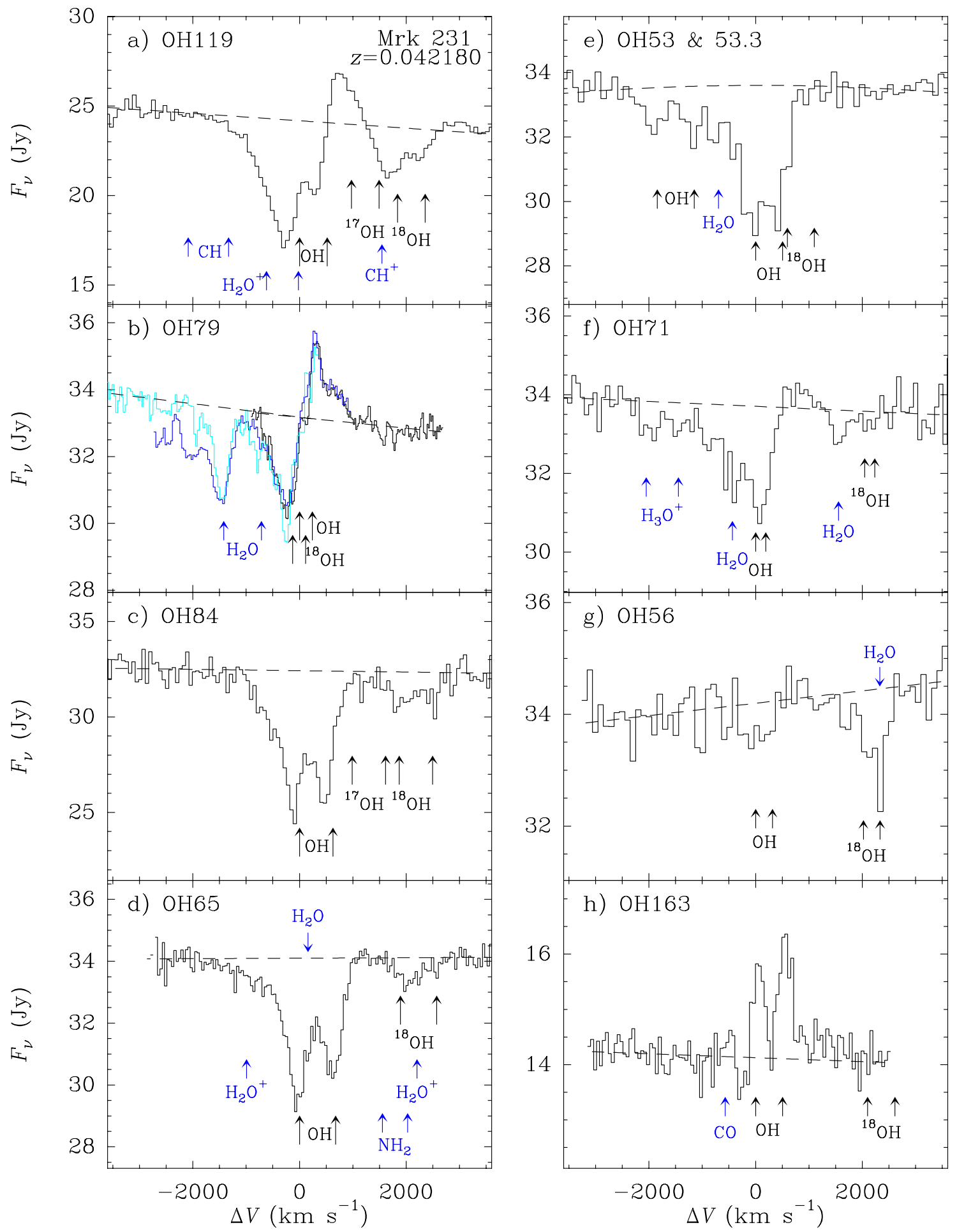

Fig. 2. Herschel/PACS spectrum of Mrk 231 around the $9 \mathrm{OH}$ doublets, with the adopted baselines (dashed lines). The velocity scale is relative to the blue $\Lambda$-component of each doublet and has been calculated with respect to the systemic redshift of $z=0.04218$. Potential contaminations due to other species are indicated in blue (and are discussed in the text). In panel b), the black and dark-blue OH79 spectra correspond to GT observations, while the light-blue spectrum corresponds to OT2 observations (see Table 1). In panel d), the OH65 spectrum corresponds to the average of the three brightest spaxels of the GT observations (GT-avg in Fig. 5).

\subsection{Potential contaminations}

Potential contaminations by lines of species other than $\mathrm{OH}$ are indicated in Fig. 2, and Fig. 4 compares the $\mathrm{OH} 65$ and OH84 spectra of Mrk 231, Arp 220, and NGC 4418. In OH119, the redshift component $J=7 / 2^{+}-5 / 2^{-}$of the excited
$N=3-2 \mathrm{CH}$ doublet $\left(E_{\text {lower }} \approx 105 \mathrm{~K}, 118.705 \mu \mathrm{m}\right)$ is not expected to contaminate the blueshifted $\mathrm{OH}$ absorption at $-1300 \mathrm{~km} \mathrm{~s}^{-1}$, because the blue component $J=7 / 2^{-}-5 / 2^{+}$ of the doublet is undetected. Likewise, there are no apparent features at the positions of the two indicated $\mathrm{p}-\mathrm{H}_{2} \mathrm{O}^{+}$lines $\left(4_{14}-3_{03} 7 / 2-5 / 2\right.$ and 9/2-7/2), also not detected in Arp 220 
E. González-Alfonso et al.: The Mrk 231 molecular outflow as seen in $\mathrm{OH}$

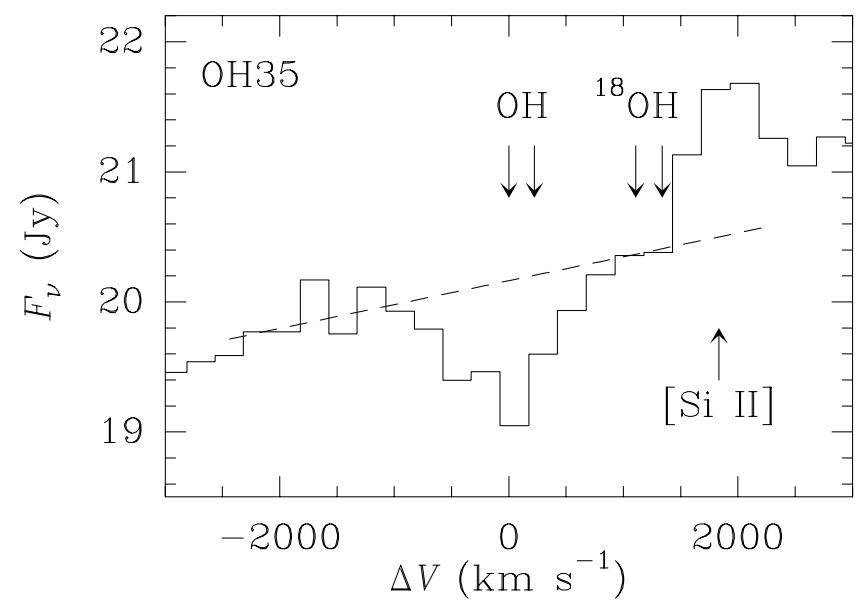

Fig. 3. Spitzer/IRS long-high (LH) spectrum around the OH35 doublet, with the adopted baseline (dashed line). The emission feature at $\sim 2000 \mathrm{~km} \mathrm{~s}^{-1}$ is the [Si II] line at $34.815 \mu \mathrm{m}$. The spectral resolution is $500 \mathrm{~km} \mathrm{~s}^{-1}$.

(González-Alfonso et al. 2013, hereafter G-A13). However, the $\mathrm{CH}^{+} 3-2$ line could be contaminating the ${ }^{18} \mathrm{OH}$ doublet (F10), as the ground transition of $\mathrm{CH}^{+}$is bright in Mrk 231 (van der Werf et al. 2010).

In the $\mathrm{OH} 79$ profile, the $\mathrm{H}_{2} \mathrm{O} 4_{23}-3_{12}$ line at $-1400 \mathrm{~km} \mathrm{~s}^{-1}$ (78.742 $\mu \mathrm{m}, E_{\text {lower }} \approx 250 \mathrm{~K}$ ) is clearly detected (F10; González-Alfonso et al. 2010, hereafter G-A10), though the prominent blueshifted absorption apparent in the GT observation is not confirmed with the OT2 observation (Table 1). On the other hand, the higher-lying $\mathrm{H}_{2} \mathrm{O} 6_{15}-5_{24}$ line at $-720 \mathrm{~km} \mathrm{~s}^{-1}$ $\left(78.928 \mu \mathrm{m}, E_{\text {lower }} \approx 600 \mathrm{~K}\right)$ was not seen in the GT observation, but a spectral feature in the OT2 observation makes the case uncertain.

In the OH84 profile, the only potential contamination is due to $\mathrm{NH}_{3}$ (Fig. 4a), which is expected to generate an absorption wing-like feature between 300 and $1300 \mathrm{~km} \mathrm{~s}^{-1}$ associated with the $(6, K) a-(5, K) s$ lines (G-A12). However, from the uncontaminated $(6, K) s-(5, K) a \mathrm{NH}_{3}$ lines at 83.4-83.9 $\mu \mathrm{m}$, only the $(6,5) s-(5,5) a$ line is (marginally) detected, thus no significant contamination by $\mathrm{NH}_{3}$ to $\mathrm{OH} 84$ is expected.

In the OH65 profile, no spectral feature coinciding with the $\mathrm{p}-\mathrm{H}_{2} \mathrm{O}^{+} 3_{30}-2_{21} 5 / 2-3 / 2$ line at $-1000 \mathrm{~km} \mathrm{~s}^{-1}$ is found (also undetected in Arp 220, Fig. 4b). The $\mathrm{H}_{2} \mathrm{O} 6_{25}-5_{14}$ line at $\approx 160 \mathrm{~km} \mathrm{~s}^{-1}$ could contaminate the main $\mathrm{OH}$ feature (G-A12), and $\mathrm{o}-\mathrm{H}_{2} \mathrm{O}^{+} 3_{31}-2_{20} 7 / 2-5 / 2$ and $\mathrm{NH}_{2} 4_{22}-3_{13}$ are probably contributing to the ${ }^{18} \mathrm{OH}$ absorption feature.

A weak contribution by the $\mathrm{H}_{2} \mathrm{O} 5_{33}-4_{22}$ line is expected around $-700 \mathrm{~km} \mathrm{~s}^{-1}$ in the $\mathrm{OH} 53.3$ profile (Fig. 2e). The $\mathrm{OH} 71$ spectral region is complex (Fig. 2f), with possible baseline curvature and contaminations on the blue side by $\mathrm{H}_{2} \mathrm{O} 5_{24}-4_{13}$ at $-430 \mathrm{~km} \mathrm{~s}^{-1}\left(E_{\text {lower }} \approx 400 \mathrm{~K}, 71.067 \mu \mathrm{m}\right)$ and possibly $\mathrm{H}_{3} \mathrm{O}^{+} 4_{1}^{-}-3_{1}^{+}$and $4_{0}^{-}-3_{0}^{+}$(both detected in NGC 4418 and Arp 220, G-A13). These lines are expected shortward of an apparent absorption pedestal $\gtrsim 1000 \mathrm{~km} \mathrm{~s}^{-1}$ wide, to which both $\mathrm{H}_{2} \mathrm{O}$ and $\mathrm{OH}$ may be contributing. On the red side, absorption by the very high-lying $\mathrm{H}_{2} \mathrm{O}_{17}-6_{06}$ line at $1550 \mathrm{~km} \mathrm{~s}^{-1}$ is detected.

The OH56 spectrum shows strong absorption by the $\mathrm{H}_{2} \mathrm{O} \quad 4_{31}-3_{22}$ transition at $2300 \mathrm{~km} \mathrm{~s}^{-1}$. The OH163 spectrum is free of contaminations, except for the relatively weak CO (16-15) emission line. Finally, the OH35 spectrum (Fig. 3) shows the [Si II] line in emission at $\sim 2000 \mathrm{~km} \mathrm{~s}^{-1}$, precluding the measurement of the ${ }^{18} \mathrm{OH} 35$ doublet profile.

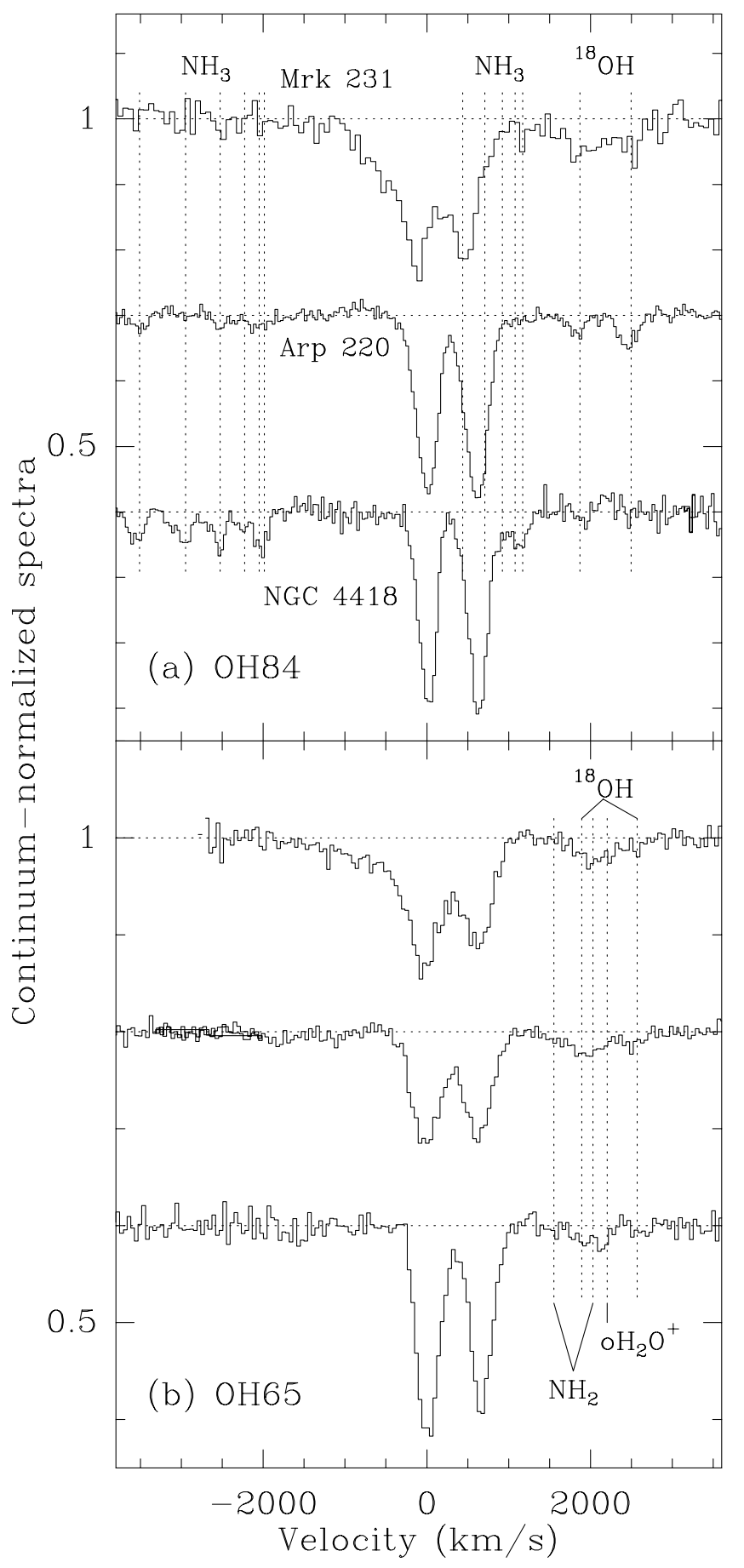

Fig. 4. Spectra around a) the $\mathrm{OH} 84$ doublet, and b) the $\mathrm{OH} 65$ doublet, in Mrk 231 (upper spectra), Arp 220 (middle), and NGC 4418 (lower). The vertical-dotted lines in a) indicate the positions of $\mathrm{NH}_{3}$ and ${ }^{18} \mathrm{OH}$ lines. The $\mathrm{NH}_{3}$ lines at $v<-2000 \mathrm{~km} \mathrm{~s}^{-1}$ are relatively strong in NGC 4418, indicating contribution by $\mathrm{NH}_{3}$ to the absorption at $v \sim 1000 \mathrm{~km} \mathrm{~s}^{-1}$. This is not the case of Mrk 231, so that there is no significant contamination at $v \sim 1000 \mathrm{~km} \mathrm{~s}^{-1}$. The ${ }^{18} \mathrm{OH} 66$ doublet in b) is complex with probable contributions by o- $\mathrm{H}_{2} \mathrm{O}^{+}$and $\mathrm{NH}_{2}$.

\subsection{Blueshifted line wings and the $\mathrm{OH} 65$ spectrum}

As mentioned in Sect. 2.1, at least the OH119, OH79, OH53.3, $\mathrm{OH} 84$, and $\mathrm{OH} 65$ doublets show high-velocity absorption wings extending up to at least $-1000 \mathrm{~km} \mathrm{~s}^{-1}$ from the blue component of the doublets; the possible $\mathrm{OH} 71$ line wing is contaminated by $\mathrm{H}_{2} \mathrm{O}$. 


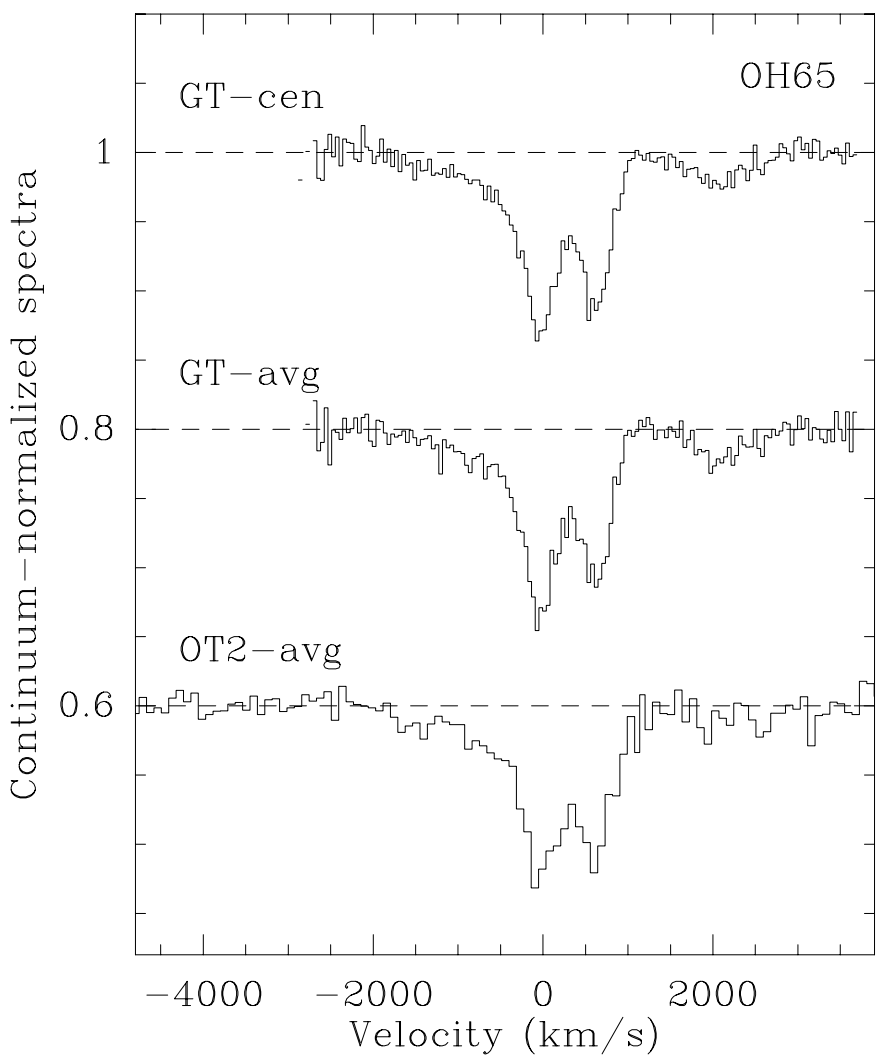

Fig. 5. OH65 doublet toward Mrk 231. Three spectra are compared: GTcen is the central spaxel of the GT observations, GT-avg is the average spectrum of the three brightest spaxels in the GT observations, and OT2-avg denotes the average spectrum of the three brightest spaxels in the OT2 observations. All three spectra show a prominent blueshifted line wing extending up to at least $\sim-1500 \mathrm{~km} \mathrm{~s}^{-1}$.

While the $\mathrm{OH} 79$ and $\mathrm{OH} 53.3$ are contaminated at velocities more blueshifted than $-1000 \mathrm{~km} \mathrm{~s}^{-1}$, the $\mathrm{OH} 119$, OH84, and OH65 doublets along the $\Pi_{3 / 2}$ ladder are uncontaminated throughout the blue range and probe the outflowing gas at the most extreme velocities. OH119 shows absorption up to $-1600 \mathrm{~km} \mathrm{~s}^{-1}$, but OH84 only up to $-1000 \mathrm{~km} \mathrm{~s}^{-1}$ with a lower $\mathrm{S} / \mathrm{N}$. Very intriguing then is the apparent strong absorption in the more excited OH65 doublet, up to velocities of at least $\sim-1500 \mathrm{~km} \mathrm{~s}^{-1}$. Figure 5 compares three spectra of the OH65 doublet. Both the GT spectra of the central spaxel (GT-cen, see Table 1) and of the three brightest spaxels (GT-avg, also shown in Fig. 2d) show the line wing covering a similar velocity range, indicating that it cannot be ascribed to pointing effects that would generate fluctuations of the continuum level. Furthermore, the OT2-avg spectrum, with a flat baseline (albeit with a lower $\mathrm{S} / \mathrm{N}$, though mostly at positive velocities), also shows the line wing with a similar velocity extent. The close agreement between the OH65 GT and OT2 high-velocity wing profiles together with the smaller extent of the OH84 profile provide strong evidence for the presence of highly excited gas at extreme velocities.

Figure 6 compares the blue line wings observed in OH119, $\mathrm{OH} 79$, $\mathrm{OH} 84$, and $\mathrm{OH} 65$ in more detail and shows the ratios of their absorption strengths. For velocities in the range -300 to $-1000 \mathrm{~km} \mathrm{~s}^{-1}$, the intensity in all spectra decreases smoothly, and the line ratios are nearly constant with the exception of the $65 / 79$, which increases significantly with increasing velocity shift. At higher velocity shifts, the $65 / 84$ and $65 / 119$ ratios jump to higher values. The limited $\mathrm{S} / \mathrm{N}$ of the $\mathrm{OH} 84$ spectrum and the

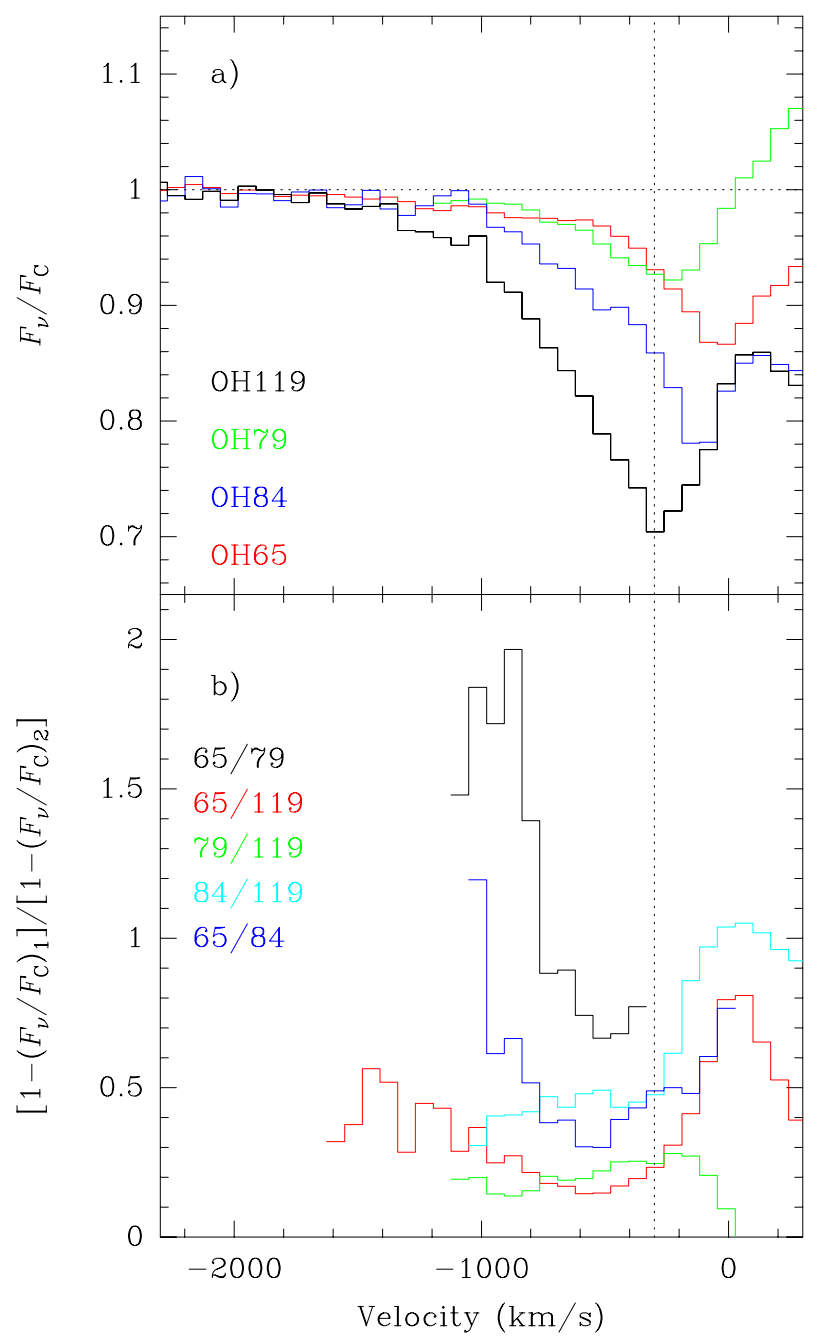

Fig. 6. a) Blueshifted line wing as observed in four $\mathrm{OH}$ doublets at 119 (black histogram), 79 (green), 65 (red), and $84 \mu \mathrm{m}$ (blue). The spectra are resampled to the velocity resolution of the OH119 spectrum. The velocity scale is relative to the blueshifted component of each doublet. b) Ratios of the absorption strengths $\left(1-F_{v} / F_{C}\right)$. The vertical dotted line at $-300 \mathrm{~km} \mathrm{~s}^{-1}$ marks the limit of the outflow region for the full set of lines.

baseline uncertainties do not allow us to establish an accurate limit for the $65 / 84$ ratio, but it is most likely $>0.5$.

\subsection{P-Cygni profiles and the role of far-IR extinction and geometry}

P-Cygni profiles are observed in at least the OH119 and OH79 doublets. The upper level of the OH119 transition is only efficiently populated from the ground-state through absorption of a $119 \mu \mathrm{m}$ photon or through a collisional event, that is, there is no efficient excitation path that involves radiative pumping to a higher-lying level and cascade down to the $\Pi_{3 / 2} J=5 / 2$ level $^{2}$. Under certain idealized conditions, this translates into a relationship between the fluxes of the absorption and emission features. Assuming spherical symmetry, a two-level system, pure

2 Pumping the $\Pi_{1 / 2} J=3 / 2,5 / 2$ levels through absorption of 53.3 and $35 \mu \mathrm{m}$ photons and subsequent decay via $\Pi_{1 / 2}-\Pi_{3 / 2} J=3 / 2,5 / 2-5 / 2$ is less efficient as the latter cross-ladder transitions are relatively weak, but can still boost the emission feature relative to the absorption one by $\sim 35 \%$. 
radiative excitation (i.e. negligible collisional excitation), and an envelope size that is much larger than the size of the continuum source (i.e. with negligible extinction of line photons emitted from behind the continuum source), then statistical equilibrium of the populations and complete redistribution in angles ensures equal number of absorption and emission events as seen by an observer that does not spatially resolve the outflow. In that case, and due to conservation of continuum photons, the outflowing gas would have the overall effect of redistributing the continuum photons in velocity space, generating a redshifted emission feature as strong as the blueshifted absorption feature ${ }^{3}$ (this is analogous to the $\mathrm{H}_{2} \mathrm{O} 6 \mu \mathrm{m}$ band in Orion BN/KL where the $\mathrm{P}$-branch is observed in emission and the R-branch in absorption, González-Alfonso et al. 1998). In Mrk 231, however, the emission feature in the OH119 doublet is five times weaker than the absorption feature, revealing $(i)$ the importance of extinction of line-emitted photons arising from the back side of the far-IR source, and/or (ii) significant departures from spherical symmetry, with the outflow mainly directed toward the observer (e.g. bipolar) and/or the receding component intrinsically less prominent than the approaching one. These conclusions are strengthened if collisions in a warm and dense region, suggested by the bright emission observed in the ground-state transitions of several species (van der Werf et al. 2010), enhance the OH119 excitation in the receding emitting gas.

Since the OH84 doublet does not show a redshifted emission feature (Figs. 2c and 4a), we argue in Sect. 3.3.1 that the circumnuclear outflowing gas does not significantly contribute to the $\mathrm{OH} 119$ emission feature (at $v>200 \mathrm{~km} \mathrm{~s}^{-1}$ from the red component, and for the reasons pointed out above), which then arises primarily from a spatially extended low-excitation component where the far-IR extinction is presumably low (Sect. 3.4). Assuming that collisional excitation of $\mathrm{OH}$ in this component is negligible (i.e. scattering of dust-emitted photons at $119 \mu \mathrm{m}$ is responsible for the observed emission), the strength of the OH119 emission feature relative to the observed continuum level then measures, under some conditions, the clumpiness of the receding gas. If the receding $\mathrm{OH}$ were intercepting the full $119 \mu \mathrm{m}$ continuum emitted toward the back $2 \pi$ sr within a velocity interval $\Delta v$, and neglecting again extinction, one would expect an emission feature as strong as $\sim 50 \%$ of $F_{119} \Delta v\left(F_{119}\right.$ is the observed continuum flux density at $119 \mu \mathrm{m})$, i.e. $\sim 6 \times 10^{3} \mathrm{Jy} \mathrm{km} \mathrm{s}^{-1}$ for $\Delta v \sim 500 \mathrm{~km} \mathrm{~s}^{-1}$ (see Fig. 2a) ${ }^{4}$. The observed emission feature, however, accounts for only $\sim 1.2 \times 10^{3} \mathrm{Jy} \mathrm{km} \mathrm{s}^{-1}$, thus suggesting a covering factor of $\sim 20 \%$ for the above conditions. If the receding $\mathrm{OH}$ is behind the source of $119 \mu \mathrm{m}$ continuum emission, only $\sim 20 \%$ of that continuum within $\Delta v=500 \mathrm{~km} \mathrm{~s}^{-1}$ is thus intercepted and reemitted by the $\mathrm{OH}$, indicating high clumpiness of the spatially extended reemitting $\mathrm{OH}$. This is consistent with the clumpiness of the outflow inferred by Aalto et al. (2012) from HCN emission.

\footnotetext{
3 In spherical symmetry, all observers located at the same distance from the source would detect exactly the same spectrum, and since we assume that there is neither cooling in the line (no collisions) nor absorption of line-emitted photons by dust, conservation of the continuum emission holds regardless of the line opacity, implying equal rates of absorption and emission events.

4 It is also assumed that the red component of the doublet dominates the emission feature and that this emission is radiatively decoupled from absorbing foreground gas; the redshifted emission due to the blue component of the doublet is cancelled or blocked by the blueshifted absorption due to the red doublet component. Note also that we use the observed line width of the emission feature as an (approximate) proxy for the velocity range within which $\tau_{\mathrm{OH} 119} \geq 1$.
}

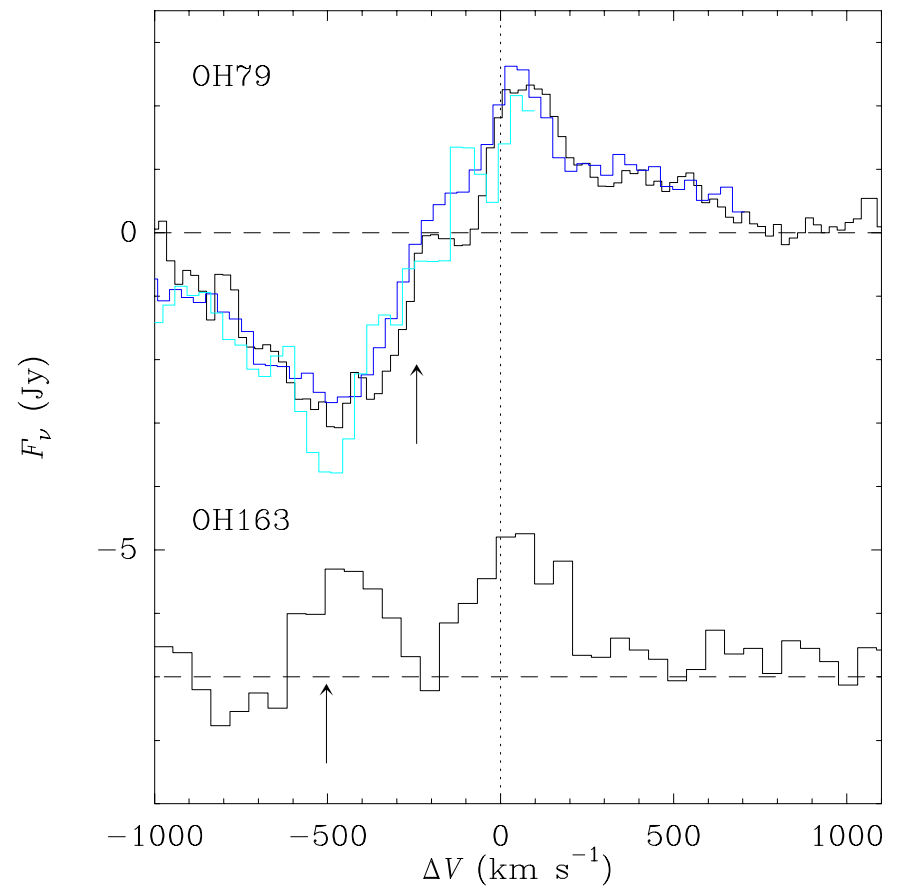

Fig. 7. Comparison between the $\mathrm{OH} 79$ and $\mathrm{OH} 163$ (vertically shifted for clarity) continuum-subtracted spectra. The velocity scale is here relative to the red $\Lambda$-component of each doublet, with the aim of directly comparing the emission features. The vertical arrows indicate the positions of the blue components of the doublets.

The ground-state $\mathrm{OH} 79$ doublet shows a relatively strong emission feature, with a flux that is nearly $75 \%$ of the absorption flux. Since extinction at $79 \mu \mathrm{m}$ is higher than at $119 \mu \mathrm{m}$, the prominent $\mathrm{OH} 79$ emission feature indicates the effect of radiative pumping through absorption of 53.3 and $35 \mu \mathrm{m}$ continuum photons, and subsequent cascading down to the ground state via the 163 and $79 \mu \mathrm{m}$ transitions (G-A12). The OH163 doublet (Fig. 2h) is indeed mostly observed in emission, qualitatively matching the pumping scheme. More quantitatively, Fig. 7 compares in detail the $\mathrm{OH} 79$ and $\mathrm{OH} 163$ profiles, with the velocity scale relative to the red component of each doublet. The flux of the $\mathrm{OH} 163$ doublet between 0 and $210 \mathrm{~km} \mathrm{~s}^{-1}$ is $\approx 400 \mathrm{Jy} \mathrm{km} \mathrm{s}^{-1}$, about $10 \%$ higher than the flux emitted in the OH79 doublet in the same velocity interval (i.e. the narrow-emission feature, $365 \mathrm{Jy} \mathrm{km} \mathrm{s}^{-1}$ ). Owing to the contribution to $\mathrm{OH} 79$ by a prominent redshifted line wing that is weak in $\mathrm{OH} 163$, the intrinsic flux of the narrow OH79 emission feature without the wing contribution is estimated to be $\sim 200 \mathrm{Jy} \mathrm{km} \mathrm{s}^{-1}$, a factor of $\sim 2$ weaker than that of the OH163. Since every $163 \mu \mathrm{m}$ line-emitted photon should be accompanied by a $79 \mu \mathrm{m}$ one (Fig. 1), but the OH79 emission is additionally boosted by direct scattering of $79 \mu \mathrm{m}$ dust-emitted photons (which does not involve emission in the $\mathrm{OH} 163$ doublet), the difference in fluxes ${ }^{5}$ indicates, regardless of geometry, that indeed significant extinction affects the narrow emission feature in the $\mathrm{OH} 79$ doublet.

The prominent redshifted line-wing observed in emission in the OH79 doublet is also remarkable, with a flux of $\approx 400 \mathrm{Jy} \mathrm{km} \mathrm{s}^{-1}$ between 210 and $800 \mathrm{~km} \mathrm{~s}^{-1}$ (Fig. 7). Some hints of emission are also found in the $\mathrm{OH} 163$ doublet at $>210 \mathrm{~km} \mathrm{~s}^{-1}$, with an uncertain $( \pm 50 \%)$ flux of $\sim 185 \mathrm{Jy} \mathrm{km} \mathrm{s}^{-1}$. This flux is weaker than that measured in the $\mathrm{OH} 79$ redshifted wing, thus

5 Fluxes in units of $\mathrm{Jy} \mathrm{km} \mathrm{s}{ }^{-1}$ are proportional to the rate of deexcitation events in the line. 
indicating that the emission in this OH79 wing is not significantly extincted and that resonant scattering of $79 \mu \mathrm{m}$ dustemitted photons probably dominates the $\mathrm{OH} 79$ wing-emission feature. It is thus plausible that the $\mathrm{OH} 79$ redshifted wing is more extended than the source of the far-IR emission. The velocity extent of the $\mathrm{OH} 79$ redshifted emission feature is similar to that of other molecular lines at millimeter wavelengths (Feruglio et al. 2010; Cicone et al. 2012; Aalto et al. 2012), suggesting a similar spatial origin.

The ground-state OH53.3 doublet (Fig. 2e) does not show an emission feature, due in part to the slightly higher chance for an $\mathrm{OH}$ molecule in the $\Pi_{1 / 2} J=3 / 2$ level to decay via the $163 \mu \mathrm{m}$ transition instead of directly emitting a $53.3 \mu \mathrm{m}$ photon (Fig. 1), but also further indicating the role of extinction. Similarly, the OH35 doublet profile (Fig. 3) only shows absorption, as expected given that essentially all molecules pumped to the upper $\Pi_{1 / 2} J=5 / 2$ level will relax by emitting a $99 \mu \mathrm{m}$ photon along the $\Pi_{1 / 2}$ ladder (instead of emitting a $35 \mu$ m photon). There is significant redshifted absorption in OH35, indicating that there is still $35 \mu \mathrm{m}$ continuum emission behind part of the receding gas.

It is then intriguing that the high-lying OH71 doublet (Fig. 2f) shows hints of redshifted emission, with a flux of $150 \mathrm{Jy} \mathrm{km} \mathrm{s}^{-1}$. The reliability of this emission feature is uncertain, however, as it shows different strengths in the central-spaxel and averaged spectra. The line should be formed very close to a warm source of far-IR radiation, which is probably optically thick at these wavelengths. If the feature is not an artifact of the baseline, inhomogeneities of the dust extinction in the nuclear region and geometry effects would be required to account for it.

\subsection{Absorption at central velocities: a very excited, quiescent gas component}

In Fig. 2, the excited $\mathrm{OH}$ lines (other than $\mathrm{OH} 163$ ) show strong absorption at central velocities, similar to NGC 4418 and Arp 220 (G-A12). This reveals the presence of a very excited, non-outflowing component in the nuclear region of Mrk 231. However, no trace of a relatively narrow absorption feature is found at central velocities in the OH119 and OH79 doublets. This is conspicuous, because extinction at $119 / 79 \mu \mathrm{m}$ would strengthen the line absorption relative to the emission feature, as argued in Sect. 2.4 for the outflowing gas. In a quiescent component, the absorption and reemission occur at the same central velocities, so that one would expect a resulting central absorption feature in spherical symmetry. The other ground-state transitions, the OH53.3 and OH35 doublets, do show strong absorption at central velocities.

The lack of measurable absorption at central velocities in the OH119 transition may be partially due to the fact that the enclosed dust is very warm $(>100 \mathrm{~K})$ and compact, thus emitting weakly at $119 \mu \mathrm{m}$ compared with the total emission at this wavelength. In this case, the OH119 absorption would be strongly diluted within the observed $119 \mu \mathrm{m}$ continuum emission, whose main contribution would arise from more extended regions devoid of quiescent $\mathrm{OH}$. In addition, collisional excitation in a warm and dense circumnuclear component would also excite the $\mathrm{OH}$ molecules to the level of near radiative equilibrium with the dust, thus producing negligible absorption. A potentially important effect is also resonant scattering of dust-emitted photons in the OH119 doublet in a flattened structure (e.g. a torus or disk) seen nearly face-on or moderately inclined, which would tend to cancel the absorption produced toward the strongest continuum source. It is worth noting that since the OH119 transition is ground-state, this process could work on spatial scales significantly larger than the region responsible for the high-excitation absorption observed at systemic velocities (see also Sect. 3.2).

While in the OH79 transition the pumping via the 53.3 and $35 \mu \mathrm{m}$ transitions enhances the reemission, in the OH53.3 doublet the upper level is higher in energy and hence more difficult to excite collisionally, and reemission is less favored because of the competing de-excitation path via the $\mathrm{OH} 163$ transition (Fig. 1).

The OH119 and OH79 profiles of Mrk 231 are in this respect very different from those observed in Arp 220, which shows in these doublets strong absorption at central velocities (G-A12). The $\mathrm{OH}$ spectra of the high-lying lines are more similar at central velocities (Fig. 4), indicating that both sources have highly excited $\mathrm{OH}$. This indicates that the components that are responsible for the ground-state absorption in Arp 220 at central velocities, that is, $C_{\text {halo }}$ and $C_{\text {extended }}$ (G-A12), are absent in Mrk 231, which is consistent with the face-on view of the disk at kpc scales.

\subsection{High $\mathrm{OH}$ optical depths}

The equivalent widths of the $\mathrm{OH} 35$ and $\mathrm{OH} 53.3$ doublets are $\approx 45$ and $\approx 120 \mathrm{~km} \mathrm{~s}^{-1}$, respectively. For optically thin absorption, ignoring reemission in the lines and assuming that the $\mathrm{OH}$ molecules are covering the whole continuum source at the corresponding wavelengths, the equivalent width of a doublet (in units of velocity) is given by

$$
W_{\mathrm{eq}}=\lambda^{3} g_{\mathrm{u}} A_{\mathrm{ul}} \frac{N_{\mathrm{OH}, \mathrm{gr}}}{8 \pi g_{\mathrm{l}}},
$$

where $\lambda$ is the wavelength, $A_{\mathrm{ul}}$ is the Einstein coefficient for spontaneous emission, $g_{\mathrm{u}}\left(g_{1}\right)$ is the degeneracy of the upper (lower) level, and $N_{\mathrm{OH}, \mathrm{gr}}$ is the $\mathrm{OH}$ column density in the two lambda-doubling states of the ground $\Pi_{3 / 2} J=3 / 2$ rotational level. Therefore, in the optically thin limit, the OH53.3-to-OH35 equivalent width ratio is $W_{\text {eq }}(\mathrm{OH} 53.3) / W_{\text {eq }}(\mathrm{OH} 35) \approx 6.4$, while the observed ratio is $\sim 3$. This indicates that opacity effects are important even in the OH53.3 doublet, which is less optically thick than the $\mathrm{OH} 79$ doublet.

Using the OH35 doublet, the lowest optical depth groundstate doublet, in Eq. (1) gives $N_{\mathrm{OH}, \mathrm{gr}} \approx 1.1 \times 10^{17} \mathrm{~cm}^{-2}$, which is a lower limit for $N_{\mathrm{OH}}$ because $(i)$ a significant fraction of molecules is in excited levels; (ii) extinction at $35 \mu \mathrm{m}$ only enables the detection of $\mathrm{OH}$ in the external layers of the continuum source; and (iii) the $\mathrm{OH}$ may not be covering the whole $35 \mu \mathrm{m}$ continuum source.

\section{7. ${ }^{18} \mathrm{OH}$}

Up to three ${ }^{18} \mathrm{OH}$ doublets are detected within the PACS range, at 120,85 , and $66 \mu \mathrm{m}$. While the ${ }^{18} \mathrm{OH} 120$ doublet may be partially contaminated by $\mathrm{CH}^{+}$, and ${ }^{18} \mathrm{OH} 66$ by $\mathrm{NH}_{2}$ and $\mathrm{H}_{2} \mathrm{O}^{+}$, the ${ }^{18} \mathrm{OH} 85$ doublet is free from contamination, with an integrated flux about five times weaker than the OH84 doublet. This confirms the strong enhancement of ${ }^{18} \mathrm{OH}$ in Mrk 231 (F10). It is also worth noting that while the $\mathrm{OH} 84$ profile shows a dip in absorption between the two lambda-doubling components, a nearly continuous bridge of absorption is seen between the ${ }^{18} \mathrm{OH}$ components (probable contamination makes the case uncertain in ${ }^{18} \mathrm{OH} 65$, where the absorption peaks in between the two lambda components). This may suggest a relative enhancement of ${ }^{18} \mathrm{OH}$ in the outflowing gas. 


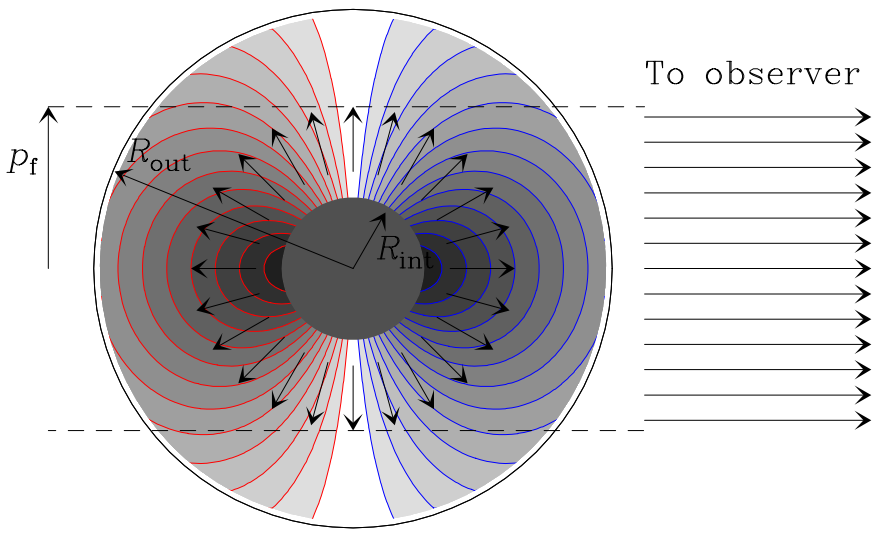

Fig. 8. Schematic representation of a given source component. It consists of $(i)$ a central source of far-IR emission characterized by its radius $\left(R_{\text {int }}\right)$, dust temperature $\left(T_{\text {dust }}\right)$, and optical depth at $100 \mu \mathrm{m}$ along a radial path $\left(\tau_{100}\right)$, and (ii) the surrounding (outflowing) gas, with external radius $R_{\text {out }}$, which is mixed with dust. The gas expands radially with a velocity field $v(r)$ and $\mathrm{H}$ nuclei density $n(r)$, such that $n_{\mathrm{OH}} \times r^{2} \times v$ is constant (i.e. the mass-outflow rate is constant). The dust in the outflowing envelope has $\tau_{50}=0.5$ along a radial path, and $T_{\text {dust }} \sim r^{-0.4}$. In some models, departures from spherical symmetry are simulated by calculating the emerging fluxes only for impact parameters lower than $p_{\mathrm{f}}$ (i.e. between the two dashed lines). The blue and red curves (and grayscale) show the isocontours of line-of-sight velocities (blue: approaching; red: receding) for a decelerating outflow; the darkest colors correspond to the highest (approaching or receding) velocities. In our best-fit models for the excited $\mathrm{OH}$ lines (the HVC component in Sect. 3.1.2), the outflowing envelope is less extended than in this representation $\left(R_{\text {out }} / R_{\text {int }} \lesssim 1.5\right)$ and is collimated $\left(p_{\mathrm{f}}<R_{\text {out }}\right)$.

\section{Models and interpretation}

\subsection{Radiative-transfer models}

\subsubsection{Overview}

To estimate the physical properties of the molecular outflow as derived from the $\mathrm{OH}$ doublets, we analyzed the $\mathrm{OH}$ line profiles and fluxes using radiative-transfer models. We used the code described in González-Alfonso \& Cernicharo (1999), which calculates in spherical symmetry the line excitation due to the dust emission and collisions with $\mathrm{H}_{2}$, and includes opacity effects (i.e. radiative trapping), non-local effects, velocity gradients, extinction by dust, and line overlaps (González-Alfonso \& Cernicharo 1997). For a given model, the code first calculates the statisticalequilibrium populations in all shells that make up the source, and then the emerging line shapes are computed, convolved with the PACS spectral resolution, and compared directly with the observations.

As shown below, at least three components (two outflowing components with different velocity fields, spatial extents, and far-IR radiation sources, and one relatively quiescent component with little or no outflowing motion, hereafter referred to as the QC) are required to obtained a reasonable match to the observed line profiles. The different components are modeled separately, and the corresponding emerging flux densities are then summed up together (i.e. it is assumed that the different components do not simultaneously overlap along the line of sight and in the projected velocity). Figure 8 depicts the generic model for a given outflowing component (the QC component described below is modeled as in G-A12). A central source of far-IR radiation is characterized by its radius $R_{\text {int }}$, dust temperature $T_{\text {dust }}$, and optical depth at $100 \mu \mathrm{m}$ along a radial path $\tau_{100}$. This is surrounded by an envelope of outflowing molecular gas with external radius $R_{\text {out }}$. The $\mathrm{OH}$ is mixed with the dust in the envelope, where $T_{\text {dust }} \sim r^{-0.4}$ (e.g. Adams 1991) and $\tau_{50}=0.5$ between $R_{\text {int }}$ and $R_{\text {out }}{ }^{6}$. The gas is outflowing in spherical symmetry with velocity and $\mathrm{H}_{2}$ density profiles $v(r)$ and $n(r)$, respectively. To decrease the number of free parameters, we imposed a constant velocity gradient (i.e. $v(r)=v_{\text {int }}+\mathrm{d} v / \mathrm{d} r\left(r-R_{\text {int }}\right)$, where $\mathrm{d} v / \mathrm{d} r=\left(v_{\text {out }}-v_{\text {int }}\right) /\left(R_{\text {out }}-R_{\text {int }}\right)$ is constant $)$ and a constant massoutflow rate (mass conservation then implies that $n_{\mathrm{OH}} \times r^{2} \times v$ is independent of $r$ ).

A constant $\mathrm{OH}$ abundance relative to $\mathrm{H}$ nuclei, $X_{\mathrm{OH}}=2.5 \times$ $10^{-6}$, was adopted (S11), as derived to within a factor of $\sim 2$ in the nuclear regions of NGC 4418 and Arp 220 (G-A12). This value is consistent with models of XDRs and CRDRs with relatively high ionization rates (e.g. Meijerink et al. 2011), that is, in the circumnuclear region of Mrk 231. In more extended regions (i.e. in the low-excitation component discussed in Sect. 3.4), the $\mathrm{OH}$ abundance may decrease depending on the reaction of $\mathrm{OH}$ with other species and photodissociation processes, or its ability to freeze-out as the outflow expands. We retain below the dependence of our mass and energy estimates on $X_{\mathrm{OH}}$ so that our results can be easily rescaled.

According to the results shown below, strict spherical symmetry is not an accurate approach in some models, and gas outflowing along two approaching and receding cocoons (i.e. with little gas expanding along the plane of sky) is favored. This is roughly simulated by including the free parameter $p_{\mathrm{f}}$, such that the emerging fluxes are calculated only for impact parameters $p<p_{\mathrm{f}}$ (i.e. for rays within the cylinder depicted with dashed lines in Fig. 8) ${ }^{7}$. Finally, the continuum-subtracted emerging profiles of a given component can be multiplied by a factor $f \lessgtr 1$, which represents either partial $\mathrm{OH}$ covering of the farIR source (i.e. a clumpy outflow, $f<1$ ), or an ensemble of independent outflows ( $f>1$, see below).

The free parameters for each component are then $R_{\text {int }}, T_{\text {dust }}$, $\tau_{100}, R_{\text {out }} / R_{\text {int }}, v_{\text {int }}, v_{\text {out }}, N_{\mathrm{OH}}, p_{\mathrm{f}} / R_{\text {out }}$, and $f$, and are listed in Table 2. The data that constrain the fit are the line profiles and fluxes of the nine $\mathrm{OH}$ doublets. The line ratios essentially depend on $T_{\text {dust }}, N_{\mathrm{OH}}$, and $R_{\text {out }} / R_{\text {int }}$, while the absolute fluxes also depend on $f R_{\text {int }}^{2}$. The radial column density of $\mathrm{H}$ nuclei in a given component is

$N_{\mathrm{H}}=X_{\mathrm{OH}}^{-1} \int_{R_{\text {int }}}^{R_{\text {out }}} n_{\mathrm{OH}}(r) \mathrm{d} r$

The mass-outflow rate per unit of solid angle is

$\frac{\mathrm{d} \dot{M}}{\mathrm{~d} \Omega}=f m_{\mathrm{H}} X_{\mathrm{OH}}^{-1} n_{\mathrm{OH}}\left(R_{\mathrm{int}}\right) R_{\mathrm{int}}^{2} v_{\mathrm{int}}$,

and the total mass-outflow rate is

$\dot{M}=4 \pi g\left(p_{\mathrm{f}}\right) \frac{\mathrm{d} \dot{M}}{\mathrm{~d} \Omega}$,

where $g\left(p_{\mathrm{f}}\right) \leq 1$ is a function that accounts for the lack of spherical symmetry $\left(g<1\right.$ for $\left.p_{\mathrm{f}}<R_{\text {out }}\right)$, and is estimated in

6 This only applies to the high-velocity component (HVC) discussed below, where a column of $N_{\mathrm{H}} \sim 10^{23} \mathrm{~cm}^{-2}$ for the outflowing shell is estimated (Table 4). A value of $\tau_{50} \approx 0.25$ across the outflowing gas is then expected (for $N_{\mathrm{H}} \sim 4 \times 10^{23} \mathrm{~cm}^{-2}$ per unit of $\tau_{50}$, G-A12). We doubled that number to roughly simulate illumination by an external radiation field and/or emitting clumps mixed with the outflowing gas, though this has a weak effect on results because the excitation is dominated by the central far-IR source.

7 Note that this is only an approximation, as the level populations are calculated in spherical symmetry. 
Table 2. Parameters for the modeling of the $\mathrm{OH}$ outflow.

\begin{tabular}{lllc}
\hline \hline Parameter & Units & Meaning & Explored range (HVC) \\
\hline$R_{\text {int }}$ & $\mathrm{pc}$ & Radius of the far-IR continuum source ${ }^{a}$ & $e$ \\
$T_{\text {dust }}$ & $\mathrm{K}$ & Dust temperature of the far-IR continuum source & $90-200$ \\
$\tau_{100}$ & & Continuum optical depth at $100 \mu$ m along a radial $\left(R_{\text {int }}\right)$ path & $0.5-4$ \\
$R_{\text {out }} / R_{\text {int }}$ & & Radius of the outflowing envelope relative to $R_{\text {int }}$ & $1.1-2.5^{f}$ \\
$v_{\text {int }}$ & $\mathrm{km} \mathrm{s}^{-1}$ & Gas velocity at $R_{\text {int }}^{b, c}$ & $1300-1700^{g}$ \\
$v_{\text {out }}$ & $\mathrm{km} \mathrm{s}^{-1}$ & Gas velocity at $R_{\text {out }}{ }^{b, c}$ & $100-400^{g}$ \\
$N_{\text {OH }}$ & $\mathrm{cm}^{-2}$ & OH column density from $R_{\text {int }}$ to $R_{\text {out }}^{b, c}$ & $(0.5-5) \times 10^{17}$ \\
$p_{\mathrm{f}}$ & $\mathrm{pc}$ & Limiting impact parameter for the calculation of emerging fluxes & $R_{\text {int }}-R_{\text {out }}{ }^{h}$ \\
$f$ & & Scaling factor ${ }^{d}$ & \\
\hline
\end{tabular}

Notes. ${ }^{(a)}$ It coincides with the inner radius of the $\mathrm{OH}$ envelope. ${ }^{(b)}$ A uniform velocity gradient is adopted, so that the velocity field is given by $v(r)=v_{\text {int }}+\mathrm{d} v / \mathrm{d} r\left(r-R_{\text {int }}\right) .{ }^{(c)}$ A constant mass-outflow rate is adopted, so that $n_{\mathrm{OH}} \times r^{2} \times v$ is independent of $r$. ${ }^{(d)}$ Representing either partial coverage by $\mathrm{OH}$ of the continuum source (a clumpy outflow, $f<1$ ), or an ensemble of independent sources $(f>1)$ ). $\dot{M}$ scales as $\sqrt{f}$. $f$ is not a fitting parameter, but indicates that the modeled source size is effective. Nevertheless, we argue in Sect. 4 that $f \sim 1$ for the QC, and in Sect. 3.3.1 that $f \gtrsim 0.45$ for the HVC. ${ }^{(e)}$ For a given model, $R_{\text {int }}$ is fixed to give the correct absolute fluxes. ${ }^{(f)}$ See Fig. $11 .{ }^{(g)}$ Accelerating velocity fields have been tried as well, but they yield poor fits to both the line profiles and the flux ratios. ${ }^{(h)} p_{\mathrm{f}}=R_{\text {out }}$ in spherical symmetry, while $p_{\mathrm{f}}=R_{\text {int }}$ simulates an outflow directed mainly toward the observer.

Appendix A. For reference, if $f=1, X_{\mathrm{OH}}^{-1} n_{\mathrm{OH}}=700 \mathrm{~cm}^{-3}$ at $r=70 \mathrm{pc}$, and $v=1000 \mathrm{~km} \mathrm{~s}^{-1}$, then $\mathrm{d} \dot{M} / \mathrm{d} \Omega \approx 90 M_{\odot} \mathrm{yr}^{-1} \mathrm{sr}^{-1}$. The momentum flux and the mechanical power, $\dot{M} v$ and $0.5 \dot{M} v^{2}$, are higher in this approach for the highest velocity gas.

The sizes we report below $\left(R_{\text {int }}, R_{\text {out }}\right)$ should be considered effective. Results identical to a given model are obtained by scaling $R_{\text {int }}$ and $R_{\text {out }}$ to higher values as $R / \sqrt{f}(f<1)$ while decreasing the densities as $n_{\mathrm{H}} \sqrt{f}$ (i.e. keeping the same column density) and decreasing the continuum-subtracted spectra as $f \times F_{\nu}$. This would approximately simulate partial covering by the outflow of the far-IR source (covering factor $f$ ). Conversely, the emerging profiles can also be interpreted as produced by an ensemble of $f$ clouds $(f>1)$ each of radius $R_{\text {int }} / \sqrt{f}$. In both cases, the mass-outflow rate scales as $\sqrt{f}$. A lower limit on $f$ is set by the condition that the modeled far-IR continuum cannot exceed the observed level. We argue below (Sects. 4 and 3.3.1) for nearly complete covering $(f \sim 0.4-1)$ for both the highexcitation quiescent component (QC) and the high-velocity component $(\mathrm{HVC})$, and give below all parameters $\left(R_{\text {int }}, R_{\text {out }}, n_{\mathrm{H}}\right)$ for $f=1$. For the low-excitation extended component (LEC) discussed below, $f \approx 0.2$ under the assumptions discussed in Sect. 2.4.

We propose in this study a set of parameters for each component that provide a reasonable match to the observed line profiles, though a more complete study will be performed in combination with the other species detected within the PACS domain. Generally speaking, the outflowing components that are highly excited (as seen in $\mathrm{OH}$ ) require compact sources (low $R_{\text {out }}$ ) and thus high mass-outflow rates.

\subsubsection{Gas components and the simplest model}

The need for several gas components is illustrated in Fig. 9 for the OH119, OH84, and OH65 doublets. Specifically, the OH84 and OH65 line profiles (Fig. 2c,d) together with their ratio (Fig. 6) are primarily used to define the gas components where $\mathrm{OH}$ is excited, while an additional low-excitation component is required to fully match the ground-state $\mathrm{OH} 119$ and OH79 doublets. One of our best-fit composite models is compared with all of the $\mathrm{OH}$ profiles in Fig. 10, where the red curves indicate the total absorption and emission as generated from all components.

- The quiescent component $(Q C)$ : the spectra of the excited lines and also the OH53.3 doublet indicate the presence of highly excited gas with the lines peaking at central velocities. The model for the QC is shown with blue curves in Figs. 9 and 10 .

- The high- and low-velocity components (HVC and LVC): in our simplest model, most of the absorption in the blueshifted line wing of the OH84 and OH65 excited doublets was simulated with a single outflowing component with a negative velocity gradient, the HVC. This component is indicated with light-blue curves in Figs. 9 and 10 and, in addition to the wing in $\mathrm{OH} 65$ and $\mathrm{OH} 84$, it contributes significantly to the absorption and emission in all other doublets, except for the high-excitation OH53 and OH56. Details of our best-fit model for the HVC are discussed and characterized in more detail in Appendix A.

The OH84 absorption at low velocities $\left(\sim 200 \mathrm{~km} \mathrm{~s}^{-1}\right)$ is not fully reproduced with the HVC alone, and additional absorption at these velocities is proposed to arise from another more extended low-velocity component (LVC). The LVC contributes slightly to the reemission in $\mathrm{OH} 119$, OH79, $\mathrm{OH} 84$, and $\mathrm{OH} 163$.

- The low-excitation component (LEC): the joint absorption and emission from the above components fails to account for the total absorption and emission observed in the groundstate $\mathrm{OH} 119$ and $\mathrm{OH} 79$ doublets, therefore the additional LEC component (green curves in Fig. 10) that produces significant absorption in these doublets but not in excited lines is required. Because of the low excitation that this component represents, it is also the most extended one, presumably tracing the outflow region probed at millimeter wavelengths by $\mathrm{CO}$ and $\mathrm{HCN}$. The parameters of this component are less constrained than those of the QC and HVC.

The inferred model parameters for the well-constrained components are listed in Table 3. We also list in Table 4 the densities, hydrogen columns, and masses associated with the QC and the $\mathrm{HVC}$, as well as the energetics that characterize the HVC.

\subsection{Quiescent Component (QC)}

The QC component is modeled as in G-A12; we adopt a "mixed" approach (i.e. the $\mathrm{OH}$ molecules and the dust are coexistent), and simulate the line broadening with a microturbulence approach $\left(v_{\text {tur }}=90 \mathrm{~km} \mathrm{~s}^{-1}\right)$. The line ratios depend on $T_{\text {dust }}$, $\tau_{100}$, and $N_{\mathrm{OH}} / \tau_{50}(\mathrm{G}-\mathrm{A} 12)$, for which the explored ranges are 
E. González-Alfonso et al.: The Mrk 231 molecular outflow as seen in $\mathrm{OH}$
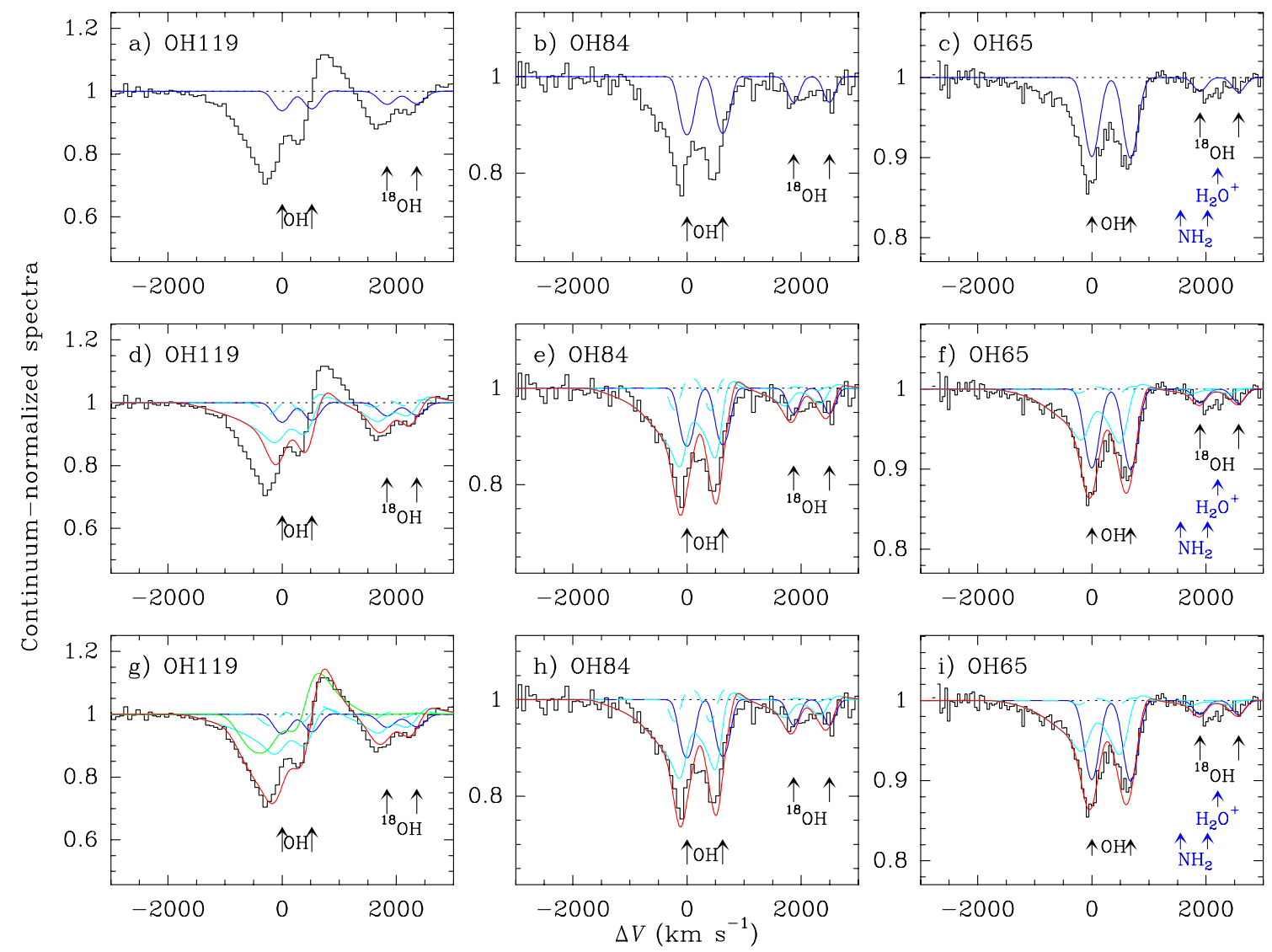

Fig. 9. Illustration of the need for several OH components in Mrk 231, as inferred from the OH119, OH84, and OH65 doublets. In panels d)-i), red curves indicate the absorption and emission by all considered components. a)-c) An outflow-free, high-excitation component (QC) generates absorption in the high-lying $\mathrm{OH}$ lines (blue curves), but cannot account for the blue wings in the three doublets or the redshifted emission in the OH119 transition. e),f) The HVC (light-blue curves) and LVC (dashed light-blue curves) reproduce the blue wings in the OH85 and OH65 doublets, but fail to account for both the full blueshifted absorption and redshifted emission in the ground-state OH119 doublet. g) A low-excitation component (LEC, green curves) is therefore required to match the ground-state OH119 (and also OH79) blueshifted absorption and redshifted emission (panel g)). The composite fit to all lines is shown in Fig. 10.

Table 3. Probable values of the parameters involved in the $\mathrm{OH}$ modeling.

\begin{tabular}{lccc}
\hline \hline Parameter & QC & HVC & LVC $^{a}$ \\
\hline$R_{\text {int }}(\mathrm{pc})^{b}$ & $55-73$ & $65-80$ & $65-80$ \\
$T_{\text {dust }}(\mathrm{K})$ & $95-120$ & $90-105$ & $\sim 90$ \\
$\tau_{100}$ & $1-3$ & $1.5-2.0$ & $\lesssim 1$ \\
$R_{\text {out }} / R_{\text {int }}$ & - & $\lesssim 1.5$ & $\sim 1.5-2$ \\
$v_{\text {int }}\left(\mathrm{km} \mathrm{s}^{-1}\right)$ & - & 1700 & $\sim 300$ \\
$v_{\text {out }}\left(\mathrm{km} \mathrm{s}^{-1}\right)$ & - & 100 & $\sim 200$ \\
$N_{\text {OH }}\left(10^{17} \mathrm{~cm}^{-2}\right)$ & $5-16^{c}$ & $1.5-3$ & $\sim 0.3$ \\
$p_{\mathrm{f}} / R_{\text {out }}{ }^{d}$ & 1 & $\sim 0.8$ & $\sim 1$ \\
\hline
\end{tabular}

Notes. Parameters for the LEC (low-excitation component) are not well constrained (see Sect. 3.4) and are omitted. (a) Uncertain parameters from the present data. ${ }^{(b)}$ For $f=1{ }^{(c)}$ Column density per unit of $\tau_{50}$ (G-A12). ${ }^{(d)} p_{\mathrm{f}} / R_{\text {out }}=1$ is a fully spherical model, while $p_{\mathrm{f}} / R_{\text {out }}<$ 1 simulates collimation in the direction of the observer.

80-130 K, 0.5-3, and $(2-20) \times 10^{17} \mathrm{~cm}^{-2}$. In our best-fit model, the line ratios are reproduced with $T_{\text {dust }}=110 \mathrm{~K}$, $\tau_{100}=1.5$, and an $\mathrm{OH}$ column of $8 \times 10^{17} \mathrm{~cm}^{-2}$ per unit of $\tau_{50}$ (blue curves in Fig. 10). For the above parameters, an effective ( $f=1$ ) radius of $R_{\text {out }} \approx 64$ pc is obtained. Similar model
Table 4. Densities, column densities, masses, and energetics.

\begin{tabular}{lcc}
\hline \hline Parameter & QC & HVC \\
\hline$n_{\mathrm{H}}\left(10^{4} \mathrm{~cm}^{-3}\right)$ & $1-2^{a}$ & $0.04-0.3^{b}$ \\
$N_{\mathrm{H}}\left(10^{24} \mathrm{~cm}^{-2}\right)$ & $1.3-4$ & $0.06-0.12$ \\
$M_{\text {gas }}\left(10^{8} M_{\odot}\right)$ & $2.5-5.0$ & $0.2-0.4$ \\
$\dot{M}\left(M_{\odot} \mathrm{yr}^{-1}\right)$ & - & $500-1200$ \\
$\dot{P}\left(10^{36} \mathrm{~g} \mathrm{~cm} \mathrm{~s}^{-2}\right)$ & - & $\sim 5-7^{c, d}$ \\
$L_{\text {mech }}\left(10^{10} L_{\odot}\right)$ & - & $\sim 6-10^{c, d}$ \\
$T_{\text {mech }}\left(10^{56} \mathrm{erg}\right)$ & - & $\sim 2-4^{d}$ \\
\hline
\end{tabular}

Notes. Assuming $X_{\mathrm{OH}}=2.5 \times 10^{-6}$ relative to $\mathrm{H}$ nuclei and $f=1$. Values scale inversely with the $\mathrm{OH}$ abundance relative to this assumed value. Only the best-constrained components, the QC and the HVC, are considered (see Sect. 3.4 for the LEC). ${ }^{(a)}$ Average density (the medium is probably clumpy). ${ }^{(b)}$ The two values correspond to the highest and lowest outflowing velocities $\left(1700\right.$ and $100 \mathrm{~km} \mathrm{~s}^{-1}$, respectively). ${ }^{(c)}$ Varies with velocity; values are given for $v=1000 \mathrm{~km} \mathrm{~s}^{-1}$. (d) Values are given for $\dot{M}=850 M_{\odot} \mathrm{yr}^{-1}$ and $R_{\text {out }} / R_{\text {int }}=1.3-1.5$.

fits are also obtained by decreasing (increasing) $T_{\text {dust }}$, and increasing (decreasing) both $N_{\mathrm{OH}} / \tau_{50}$ and $R_{\text {out }}$; the most plausible ranges are $T_{\text {dust }}=95-125 \mathrm{~K}, N_{\mathrm{OH}} / \tau_{50}=(16-5) \times 10^{17} \mathrm{~cm}^{-2}$, and $R_{\text {out }}=73-55 \mathrm{pc}$, respectively (Table 3 ). For a hydrogen column of $N_{\mathrm{H}}=4 \times 10^{23} \mathrm{~cm}^{-2}$ per unit of $\tau_{50}$ (G-A12), 

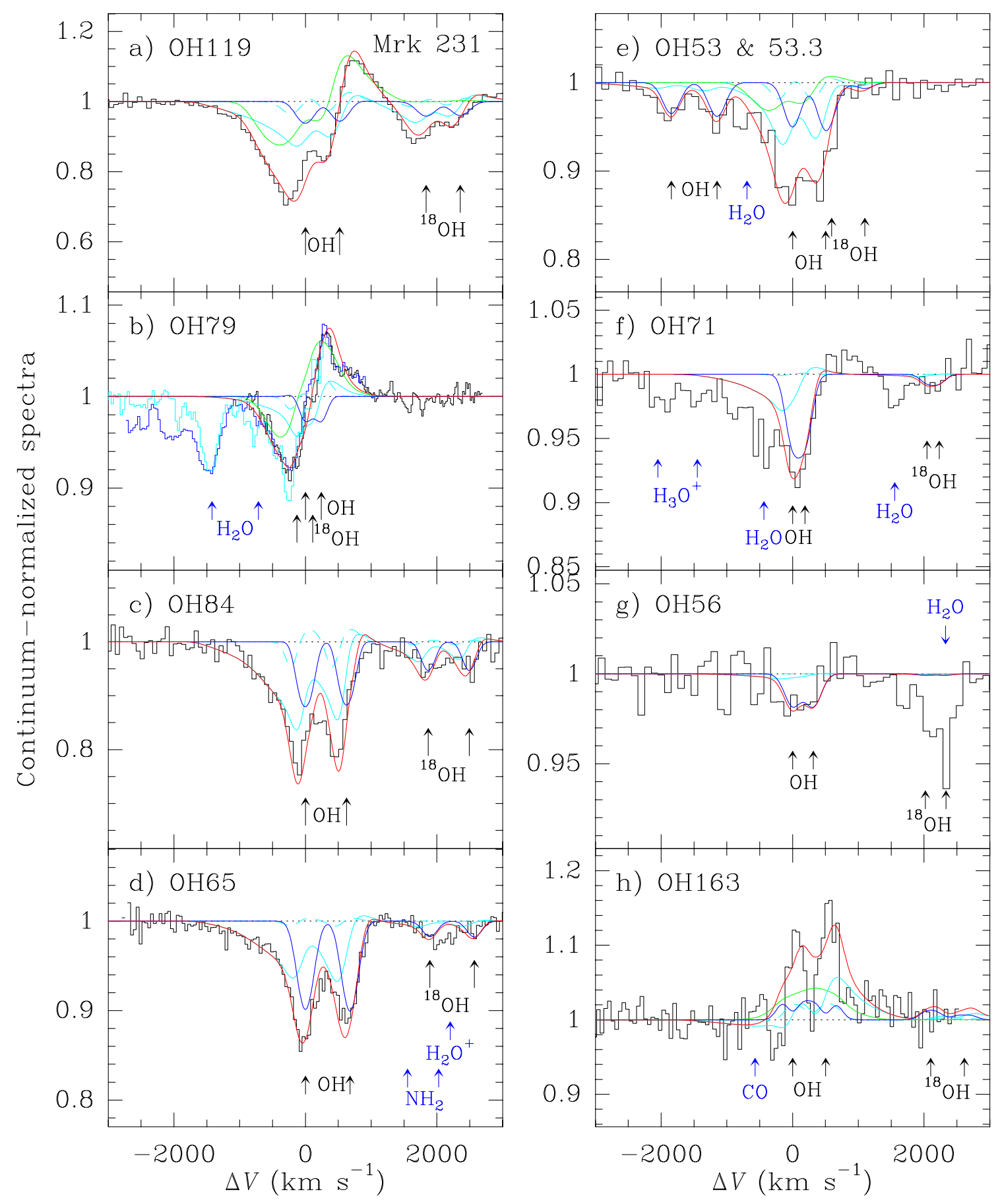

Fig. 10. Model fit for the $\mathrm{OH}$ doublets in Mrk 231. The blue, light-blue, dashed light-blue, and green curves show the contributions by the quiescent component (QC), high-excitation outflow component (HVC), low-velocity component (LVC), and low-excitation component (LEC), respectively. Red is the total emission and absorption due to all components. In this specific model, the parameters for the $\mathrm{HVC}$ are $T_{\mathrm{dust}}=105 \mathrm{~K}, R_{\mathrm{int}}=74 \mathrm{pc}$, $R_{\text {out }} / R_{\text {int }}=1.3, N_{\mathrm{OH}}=1.6 \times 10^{17} \mathrm{~cm}^{-2}, v_{\text {int }}=1700 \mathrm{~km} \mathrm{~s}^{-1}, v_{\text {out }}=100 \mathrm{~km} \mathrm{~s}^{-1}$, and $p_{\mathrm{f}}=1.15 \times R_{\text {int }}$, and the mass-outflow rate per unit of solid angle is $\mathrm{d} \dot{M} / \mathrm{d} \Omega \approx 100 M_{\odot} \mathrm{yr}^{-1} \mathrm{sr}^{-1}$.

$N_{\mathrm{OH}} / \tau_{50}=8 \times 10^{17} \mathrm{~cm}^{-2}$ gives $X_{\mathrm{OH}}=2 \times 10^{-6}$, in close agreement with our adopted value. Both $T_{\text {dust }}$ and $N_{\mathrm{OH}} / \tau_{50}$ are similar to those inferred in the nuclear region of Arp 220 (G-A12). The total $\mathrm{OH}$ column along a radial path (assuming uniform conditions) is $\sim 4 \times 10^{18} \mathrm{~cm}^{-2}$. The continuum optical depth is similar to that of the HVC (Sect. 3.3), $\tau_{100}=1.5$, and corresponds to $N_{\mathrm{H}} \sim 2 \times 10^{24} \mathrm{~cm}^{-2}$. Values of $\tau_{100}>3$ would produce an absorption feature in the OH163 doublet at central velocities ${ }^{8}$,

\footnotetext{
8 The $\mathrm{H}_{2} \mathrm{O}$ absorption lines, however, favor $\tau_{100} \sim 3$, as will be reported in a future work.
}

while $\tau_{100}<1$ would fail in reproducing the strong absorption in the $\mathrm{OH} 71$ doublet at central velocities.

The QC is significantly smaller (for $f=1$ ) and most likely warmer than the "warm component" needed to reproduce the $\mathrm{H}_{2} \mathrm{O}$ lines detected in Mrk 231 with SPIRE (95 K and $120 \mathrm{pc}$, G-A10). Preliminary models for $\mathrm{H}_{2} \mathrm{O}$ indicate, however, that the $\mathrm{H}_{2} \mathrm{O}$ absorption lines are fitted with a model source size as small as that of the QC. This behavior suggests that while the $\mathrm{H}_{2} \mathrm{O}$ absorption is primarily produced in front of the compact far-IR optically thick cores, efficient $\mathrm{H}_{2} \mathrm{O}$ submillimeter line emission is 
generated in the surrounding more extended regions with lower extinction, but with a far-IR radiation density sufficiently high to produce the observed excitation. The relationship between the $\mathrm{H}_{2} \mathrm{O}$ absorption and emission lines will be explored in a future study.

To avoid too strong absorption at central velocities in the ground $\mathrm{OH} 119$ and $\mathrm{OH} 79$ doublets, significant collisional excitation with a density of $2.5 \times 10^{6} \mathrm{~cm}^{-3}$ (much higher than the average density of $(1-2) \times 10^{4} \mathrm{~cm}^{-3}$, Table 4) was used. As discussed in Sect. 2.5, however, significant departures from spherical symmetry (i.e. reemission from a flattened and possibly more extended structure viewed nearly face-on or with moderate inclination) could also account for the weakness of these lines. Efficient reemission at central velocities could take place in the surrounding region primarily responsible for the $\mathrm{H}_{2} \mathrm{O}$ submillimeter emission, or at even the larger scales of the face-on disk (Downes \& Solomon 1998). In any case, the above density is effective because collisional excitation with electrons and atomic hydrogen in an environment with a high-ionization fraction would additionally relax the required value. Nevertheless, the density cannot be lower than several $\times 10^{4} \mathrm{~cm}^{-3}$, indicating that the QC is clumpy. In these effective models, collisional excitation primarily affects the ground-state OH119 and OH79 transitions.

\subsection{High- and low-velocity components (HVC and LVC)}

In our simplest approach, the observed absorption in the line wings of the excited OH84 and OH65 doublets at $v<$ $-400 \mathrm{~km} \mathrm{~s}^{-1}$ are simulated with a single outflow component, the HVC. It is characterized by a decelerating velocity field with $v_{\text {int }}=1700 \mathrm{~km} \mathrm{~s}^{-1}$ and $v_{\text {out }}=100 \mathrm{~km} \mathrm{~s}^{-1}$, with hydrogen column densities in velocity intervals of $100 \mathrm{~km} \mathrm{~s}^{-1}$, as shown in Fig. A.1a. We used two values for $T_{\text {dust }} ; 105 \mathrm{~K}$, which is close to the value used for the QC, and $90 \mathrm{~K}$, closer to the value used for the warm component in G-A10. A moderately high $\tau_{100}=1.5$ is favored, motivated by the blueshifted absorption seen in the OH163 doublet.

\subsubsection{Column density and spatial scale of the HVC component}

How extended is the outflowing gas in the HVC (as seen in $\mathrm{OH})$ as compared with the source of far-IR emission that excites the OH? In Fig. 11a, the OH65-to-OH84 equivalent-width ratio (solid curves) is plotted as a function of the adopted $R_{\text {out }} / R_{\text {int }}$ for two combinations of $\left(T_{\text {dust }}, N_{\mathrm{OH}}\right)$. The observed ratio $(\approx 0.4-0.5)$ can be reproduced either with $\left(T_{\text {dust }}=105 \mathrm{~K}, N_{\mathrm{OH}}=1.6 \times\right.$ $\left.10^{17} \mathrm{~cm}^{-2}\right)$, or with $\left(T_{\text {dust }}=90 \mathrm{~K}, N_{\mathrm{OH}}=3.2 \times 10^{17} \mathrm{~cm}^{-2}\right)$, as long as the thickness of the outflowing shell is small in comparison to the radius of the far-IR source (i.e., $R_{\text {out }} / R_{\text {int }} \lesssim 1.5$ ).

In a more extended outflow $\left(R_{\text {out }} / R_{\text {int }}>1.5\right)$ the $\mathrm{OH}$ becomes less excited, the predicted $\mathrm{OH} 65 / \mathrm{OH} 84$ ratio drops, and higher columns are then required. However, an extended HVC would have an observable effect on the line shapes. In spherical symmetry, one would expect an emission feature at redshifted velocities (see Fig. 11c), arising from the limb of the farIR source where the continuum optical depth is relatively low (for impact parameters $p$ higher than $R_{\text {int }}$, Fig. 8). This modeled emission feature, especially prominent in the OH84 doublet, is not seen in the spectra. Reemission in $\mathrm{OH} 84$ is not occurring at high velocities, that is, not in the HVC (for the LVC, see below), indicating that the projected surface where reemission
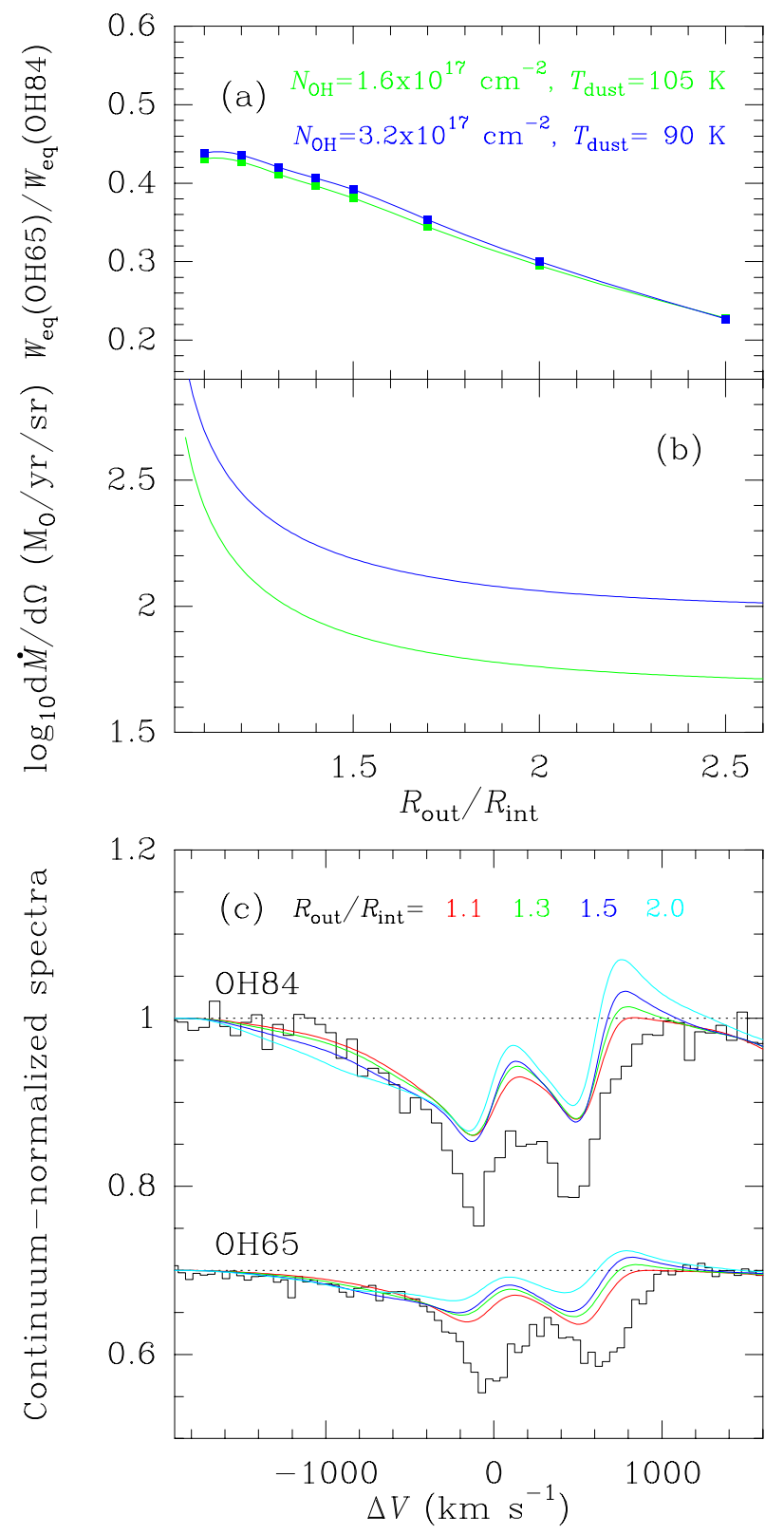

Fig. 11. Model results for $\mathrm{OH} 84$ and $\mathrm{OH} 65$ in the HVC component. a) Ratio of the OH65-to-OH84 equivalent widths in the blueshifted wing as a function of the thickness of the outflowing shell, $R_{\text {out }} / R_{\text {int }}$. $N_{\mathrm{OH}}$ and $T_{\text {dust }}$ are given by $1.6 \times 10^{17} \mathrm{~cm}^{-2}, 105 \mathrm{~K}$ (green curve), and $3.2 \times 10^{17} \mathrm{~cm}^{-2}, 90 \mathrm{~K}$ (blue curve), yielding very similar results. Other model parameters are $\tau_{100}=1.6, v_{\text {int }}=1700 \mathrm{~km} \mathrm{~s}^{-1}$, and $v_{\text {out }}=100 \mathrm{~km} \mathrm{~s}^{-1}$. b) Corresponding mass-outflow rates per unit solid angle for full coverage of the far-IR source ( $f=1$, corresponding to $R_{\text {int }}=75 \mathrm{pc}$; see also Eq. (A.1) and Table 3). The assumed $\mathrm{OH}$ abundance relative to $\mathrm{H}$ nuclei is $X_{\mathrm{OH}}=2.5 \times 10^{-6}$. c) Observed (black histograms) and predicted $\mathrm{OH} 84$ and $\mathrm{OH} 65$ line profiles $\left(N_{\mathrm{OH}}=\right.$ $3.2 \times 10^{17} \mathrm{~cm}^{-2}$ ) for $R_{\text {out }} / R_{\text {int }}=1.1$ (red curves), 1.3 (green), 1.5 (blue), and 2.0 (light-blue).

by the excited $\mathrm{OH}$ is generated is not significantly larger than the surface where the absorption is produced. This suggests that either the HVC is compact around the optically thick far-IR continuum source, or that the outflow is collimated ( $p_{\mathrm{f}} \sim R_{\text {int }}$, Fig. 8), flowing just toward (and possibly in the opposite direction of) the observer. In an extended/collimated HVC, however, the line shapes would differ significantly from the observations. The covering factor as a function of the line-of-sight velocity 
would have little contrast between moderately low and high velocities, thus predicting relatively flat blueshifted line wings that are hardly compatible with the observed steep OH84 blueshifted wing. The model fit for an extended outflow grossly overpredicts the OH84 absorption at $\sim-1000 \mathrm{~km} \mathrm{~s}^{-1}$ relative to OH65 (light-blue curve in Fig. 11b). While some degree of collimation is probably present (see Sect. 2.4 and below), the observed line shapes and high $\mathrm{OH}$ excitation argue in favor of a component where the high-velocity $\mathrm{OH}$ gas is piled up into a relatively narrow region, tracing excited gas blowing out (along with the warm dust) from the warm far-IR continuum source against which we see the $\mathrm{OH}$ absorption. We therefore favor $R_{\text {out }} / R_{\text {int }} \lesssim 1.5$.

For the case of full coverage of the far-IR source $(f=1)$, the size of the far-IR source required to reproduce the absolute fluxes is $R_{\text {int }} \sim 65-80 \mathrm{pc}$, and the outflow size (diameter) is $\sim 200$ pc. A lower limit, $f \gtrsim 0.45$, is set by the constraint that the continuum flux density at $30-50 \mu \mathrm{m}, 7.5-12.5 \mathrm{Jy}$, cannot exceed the observed continuum level, implying a physical size not higher than $1.5 \times R_{\text {int }}$ and an outflow size of up to $\sim 300 \mathrm{pc}$. The size of the far-IR continuum source behind the HVC is slightly larger than but similar to that of the QC component, suggesting that the outflow fully covers the QC.

The OH84 and OH65 blueshifted wings can be reproduced almost equally well with $\left(T_{\text {dust }}=105 \mathrm{~K}, N_{\mathrm{OH}}=1.6 \times 10^{17} \mathrm{~cm}^{-2}\right)$, and with $\left(T_{\text {dust }}=90 \mathrm{~K}, N_{\mathrm{OH}}=3.2 \times 10^{17} \mathrm{~cm}^{-2}\right)$, illustrative of degeneracies in the models when constrained only by these two transitions. However, significant differences between the two models are seen especially in the ground-state OH79 and OH53.3 doublets, which are overpredicted by the high columndensity solution. On the other hand, the high strength of the ${ }^{18} \mathrm{OH} 85$ doublet favors high $\mathrm{OH}$ columns (Sect. 3.6), so that the column density is probably within the range $N_{\mathrm{OH}}=(1.5-3.0) \times$ $10^{17} \mathrm{~cm}^{-2}$.

Even with a compact shell with $R_{\text {out }} / R_{\text {int }}=1.3$, our spherical models overpredict the reemission at redshifted velocities in the OH84 doublet profile, so that $p_{\mathrm{f}} / R_{\text {out }}<1$ is favored $(\sim 0.8$, Table 3 ). As a consequence of the compactness and collimation, the predicted redshifted reemission in the HVC component of the OH119 and OH79 doublets is weak (see also Fig. 9), so the observed emission features remain as residuals, and we attribute them to more spatially extended components, that is, the LVC and mostly the LEC discussed below.

\subsubsection{LVC component}

In most of the generated models for the HVC, the OH84 absorption at low blueshifted velocities $\left(200-300 \mathrm{~km} \mathrm{~s}^{-1}\right)$ is underpredicted. Broadening of the absorption by the QC due to rotation of the circumnuclear structure (torus or thick disk) could account for some of this missing absorption. However, the responsible gas is less excited than in the HVC, as little additional absorption in $\mathrm{OH} 65$ is required for a good fit of the profile. Therefore, we tentatively associate this absorption with an increase of the covering factor of the continuum by the $\mathrm{OH}$ at these velocities. Even if this additional low-velocity absorption is probably produced by a spatial extension of the HVC in the plane of sky, with velocities lower than predicted by the HVC, it is modeled in our spherically symmetric models by means of a separate component, the LVC (dashed light-blue lines in Fig. 10). The LVC is more extended than the HVC, generating some reemission in $\mathrm{OH} 119, \mathrm{OH} 79, \mathrm{OH} 84$, and OH163. In general, the inferred parameters of the LVC are rather uncertain because the associated absorption overlaps with that produced by the HVC; we modeled it with $R_{\text {out }}=150 \mathrm{pc}$ and $N_{\mathrm{OH}}=3 \times 10^{16} \mathrm{~cm}^{-2}$. This is a minor component, contributing little to the observed spectra and only at low velocities.

\subsubsection{Velocity field?}

The $\mathrm{OH} 65 / \mathrm{OH} 84$ ratio in the blueshifted line wing is relatively flat for $v>-900 \mathrm{~km} \mathrm{~s}^{-1}$, and tends to increase (or at least remain similar) with higher velocity shifts. This dependence provides clues about the relative location of the gas at different velocities with respect to the source of excitation. If the $\mathrm{OH}$ excitation were independent of velocity, saturation of the OH84 doublet at low velocities (Fig. A.1c) would enhance the OH65/OH84 ratio at these velocities. This is contrary to the observed trend, suggesting that the $\mathrm{OH}$ gas with the highest velocity shift is more excited than the low-velocity outflowing gas. The increasing excitation with increasing velocity shift is, in our models, generated by locating the higher velocity gas closer to the far-IR exciting source than the lower velocity gas (Fig. A.1b), thus suggesting an overall decelerating velocity field. We also tried to model the HVC with accelerated velocity flows, but found that the modeled line shapes and line flux ratios were inconsistent with observations. In our models, the LVC is more extended and less excited than the HVC, supporting the same decelerating scenario. We note, however, that this solution relies on our simple spherical geometry (where the successive shells are concentric) and may not be unique; for example, the high- and low-velocity gas may be flowing from different regions of a circumnuclear torus or disk, characterized by different $T_{\text {dust }}$ and possibly with different projection effects as the outflow widens. Nevertheless, some deceleration is most likely taking place because $\mathrm{CO}$ and $\mathrm{HCN}$, which trace larger regions, show wings up to a velocity of $\sim 800 \mathrm{~km} \mathrm{~s}^{-1}$ from the line center (Feruglio et al. 2010; Cicone et al. 2012; Aalto et al. 2012), significantly lower than OH. Spoon \& Holt (2009) also inferred a decelerating velocity field from the ionized gas outflows traced by the [NeII], [NeIII] and [NeV] lines in a sample of ULIRGs, though not in Mrk 231; the fine-structure mid-IR lines trace an outflow on a significantly smaller spatial scale, however.

The very strong velocity gradient used in our model fits, with the gas velocities varying from 1700 to $100 \mathrm{~km} \mathrm{~s}^{-1}$ in a relatively short path ( $\$ 40 \mathrm{pc}$ ), may be indicative of high clumpiness and turbulence within the flow, but also favors a nonconcentric origin of gas at different velocities. Nevertheless, strong shocks in swept-up gas of high density and column could in principle produce a strong deceleration of the previously accelerated gas. It is also worth noting that the LEC described below also indicates the presence of high-velocity gas (up to $\sim 900 \mathrm{~km} \mathrm{~s}^{-1}$ ) detached from the nuclear region, representing high-velocity gas that escapes from the nuclear region along paths of least resistance.

\subsubsection{Energetics}

For $N_{\mathrm{OH}}=1.5 \times 10^{17} \mathrm{~cm}^{-2}, R_{\text {int }}=70 \mathrm{pc}$, and $R_{\text {out }} / R_{\text {int }} \lesssim 1.5$, the mass-outflow rate per solid angle in the direction of the observer (Eq. (A.1)) associated with the HVC is $\mathrm{d} \dot{M} / \mathrm{d} \Omega \gtrsim$ $68 \sqrt{f}\left(2.5 \times 10^{-6} / X_{\mathrm{OH}}\right) M_{\odot} \mathrm{yr}^{-1} \mathrm{sr}^{-1}$. In spherical symmetry, this corresponds to $\dot{M} \gtrsim 850 g\left(p_{\mathrm{f}}\right) \sqrt{f} M_{\odot} \mathrm{yr}^{-1}$, but we favor a collimated outflow $\left(p_{\mathrm{f}} \sim R_{\text {int }}\right)$ such that, for $R_{\text {out }} / R_{\text {int }}=1.5$, the anisotropy function $g\left(p_{\mathrm{f}}\right)$ may be as low as $\sim 0.4$ (increasing steeply for smaller sizes). At least several $\times 10^{2} M_{\odot} \mathrm{yr}^{-1}$, and possibly $\sim 10^{3} M_{\odot} \mathrm{yr}^{-1}$, are inferred locally in the circumnuclear region of Mrk 231 (Table 4). However, it is just the compact 
nature of the HVC gas that may suggest a non-steady flow, leaving open the possibility of intermittency.

In our prescription, the momentum flux increases with gas velocity and is given by $\dot{P}=5.3 \times$ $10^{36}\left(\dot{M} / 850 M_{\odot} \mathrm{yr}^{-1}\right)\left(v / 10^{3} \mathrm{~km} \mathrm{~s}^{-1}\right)\left(2.5 \times 10^{-6} / X_{\mathrm{OH}}\right) \mathrm{g} \mathrm{cm} / \mathrm{s}^{2}$, or $\sim 15 L_{\mathrm{AGN}} / c$, adopting $L_{\mathrm{AGN}}=2.8 \times 10^{12} L_{\odot}$ (Veilleux et al. 2009). The corresponding mechanical luminosity is $L_{\text {mech }} \sim 6 \times 10^{10} L_{\odot}$. The uncertainty in these parameters $(\dot{M}, \dot{P}$, and $\left.L_{\text {mech }}\right)$ is as high as a factor $\sim 3$ mainly because of geometry effects and the uncertainty in the $\mathrm{OH}$ abundance. While our estimates for the rates $\left(\dot{M}, \dot{P}\right.$, and $\left.L_{\text {mech }}\right)$ are roughly consistent with those inferred from $\mathrm{CO}$ by Feruglio et al. (2010), our integral values $\left(M_{\text {gas }}\right.$ and $\left.T_{\text {mech }}\right)$ are much lower due to the compactness of the HVC.

\subsection{Low-excitation component (LEC)}

While the joint emission/absorption from the above three (QC, HVC, and LVC) components properly describes the observed absorption in the excited doublets, the ground-state OH119 and OH79 lines remain underpredicted. An additional low-excitation component (LEC) that accounts for the remaining OH119 and OH79 flux, but does not significantly contribute to the excited $\mathrm{OH}$ doublets, was therefore included in the model. The LEC is expected to be more spatially extended than the source of farIR emission so that the $\mathrm{OH}$ molecules remain essentially in the ground-state, and is also expected to be primarily responsible for the emission features detected in $\mathrm{OH} 119$ and $\mathrm{OH} 79$ at redshifted velocities. Because this component is traced by the ground-state doublets, no additional constraints on the spatial extent can be inferred from the $\mathrm{OH}$ data. Nevertheless, it is reasonable to assume that the LEC probes the relatively extended outflowing emission measured at millimeter wavelengths (Feruglio et al. 2010; Cicone et al. 2012; Aalto et al. 2012).

Figure 12a shows the OH119 and OH79 profiles after subtracting the modeled emission of the QC+HVC+LVC (i.e. the modeled components for the excited $\mathrm{OH}$ ). If our composite model for the excited $\mathrm{OH}$ is sufficiently accurate, the profiles in Fig. 12a thus isolate the contribution by the LEC to the observed profile. We note, however, that the emission at $v \sim 0-200 \mathrm{~km} \mathrm{~s}^{-1}$ may still have a substantial contribution from circumnuclear gas. In OH119, even collisional excitation in a warm, dense region may take place at these moderately redshifted velocities where the CO 16-15 line appears to peak (Fig. 10h) ${ }^{9}$. The LEC contribution in Fig. 12a is thus tentative at low velocities. It is nevertheless interesting that the OH119/LEC shows a nearly symmetric line shape with an emission feature only $20 \%$ weaker than the absorption feature, and with similar velocity extents on the blue and red sides. Within the model uncertainties and according to the discussion in Sect. 2.4, this result is consistent with a roughly spherical distribution of the LEC with negligible extinction effects at $119 \mu \mathrm{m}$, indicating a wide opening angle of the flow at the corresponding spatial scales.

Since detection of OH79 in the LEC ensures that the OH119 doublet is optically thick, the absorption of the LEC OH119 normalized spectrum directly gives the covering factor at each lineof-sight blueshifted velocity $\left(f_{v}=1-F_{v} / F_{c}\right.$, where $F_{v} / F_{c}$ is the continuum-normalized spectrum in Fig. 12a), uncorrected for

\footnotetext{
9 The ground-state lines of $\mathrm{OH}^{+}, \mathrm{CH}^{+}$, and $\mathrm{HF}$ are all detected in emission (van der Werf et al. 2010), indicating the importance of collisional excitation in these ground transitions; the observed emission in $\mathrm{OH} 119$ at central velocities may also have a substantial contribution from collisionally excited gas in the same warm/dense region.
}

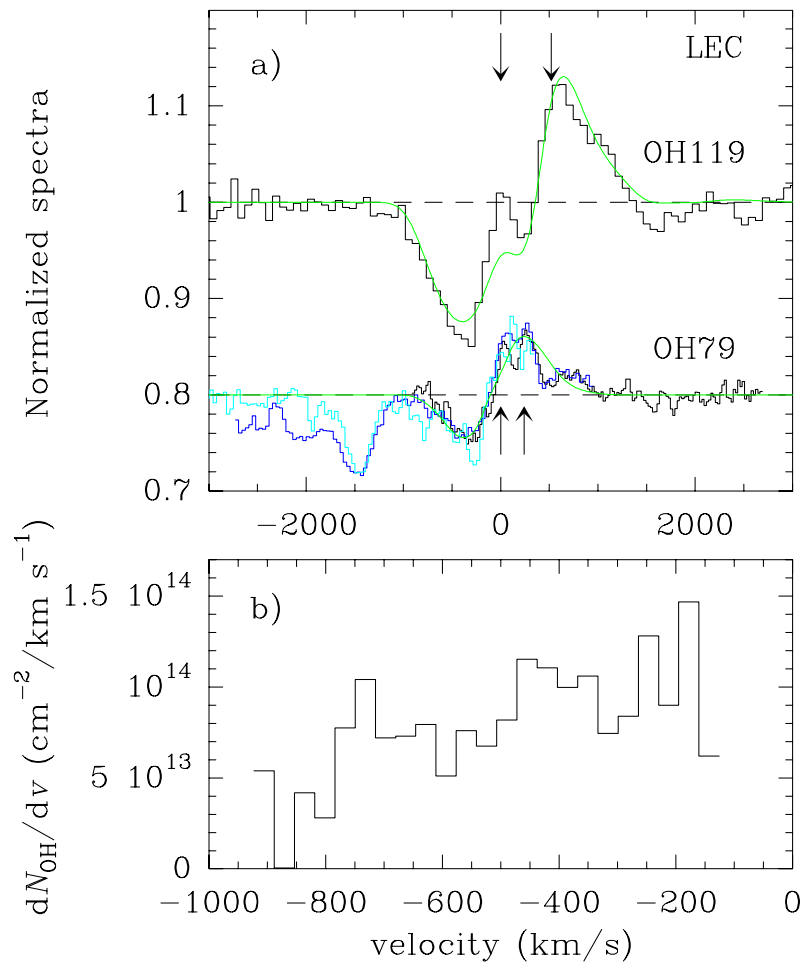

Fig. 12. a) $\mathrm{OH} 119$ and $\mathrm{OH} 79$ doublets after subtracting the model for the $\mathrm{QC}+\mathrm{HVC}+\mathrm{LVC}$, thus tentatively isolating the contribution of the low-excitation component (LEC) to the absorption and emission. The green curves show our simple spherically symmetric model for the LEC (Sect. 3.4). b) Inferred OH column density of the LEC per unit of lineof-sight velocity interval across the blue absorption wing after correcting for the covering factor at each velocity, but not correcting for the reemission in the lines (see text). The integral gives a total LEC column of $N_{\mathrm{OH}} \approx 7 \times 10^{16} \mathrm{~cm}^{-2}$, in agreement with the detailed models.

the reemission in the line. The $\mathrm{OH}$ column per unit of velocity interval was estimated from the $\mathrm{OH} 79$ doublet (also uncorrected for the line reemission), and is shown in Fig. 12b. The integral of this spectrum gives $N_{\mathrm{OH}} \approx 7 \times 10^{16} \mathrm{~cm}^{-2}$, in agreement with the model for the LEC discussed below that accurately takes into account the reemission in both doublets.

Models for the LEC have significant degeneracies because of $(i)$ the uncertainty in the shape and strength of the far-IR continuum field as seen by the absorbing and emitting $\mathrm{OH}$, and (ii) the lack of constraints on the spatial scale. Our simple model for the LEC (green curves in Figs. 12a and 10a,b,e, and h) assume the following: $(i)$ the LEC surrounds the whole source of far-IR emission, which is described by a spherical source with $R_{\text {int }}=490 \mathrm{pc}, T_{\text {dust }}=55 \mathrm{~K}$, and $\tau_{100}=0.5$ to nearly fit the observed continuum between 50 and $130 \mu \mathrm{m}$; (ii) we adopted an external radius of $R_{\text {out }}=800 \mathrm{pc}$ (corresponding to the $\sim \mathrm{e}^{-1}$ level of the $F W H M=1.3 \mathrm{kpc}$ CO $1-0$ line region, Cicone et al. 2012 $)^{10}$. Finding a reasonable match to the doublet shapes again requires a decelerating flow, with $v_{\text {int }}=900 \mathrm{~km} \mathrm{~s}^{-1}$ and $v_{\text {out }}=200 \mathrm{~km} \mathrm{~s}^{-1}$. The gas velocity fields as derived from OH119 in other sources will be explored in a future work.

The model fit in Fig. 12a uses $N_{\mathrm{OH}} \approx 6.3 \times 10^{16} \mathrm{~cm}^{-2}$ (in close agreement with Fig. $12 \mathrm{~b}$ ), $p_{\mathrm{f}}=R_{\text {out }}$ (i.e. strict spherical

\footnotetext{
${ }^{10}$ Note, however, that $\mathrm{OH}$ can potentially trace regions more extended than those traced by $\mathrm{CO}$, because $\mathrm{CO}$ requires a minimum density to be collisionally excited while $\mathrm{OH}$ only needs the available far-IR radiation field.
} 


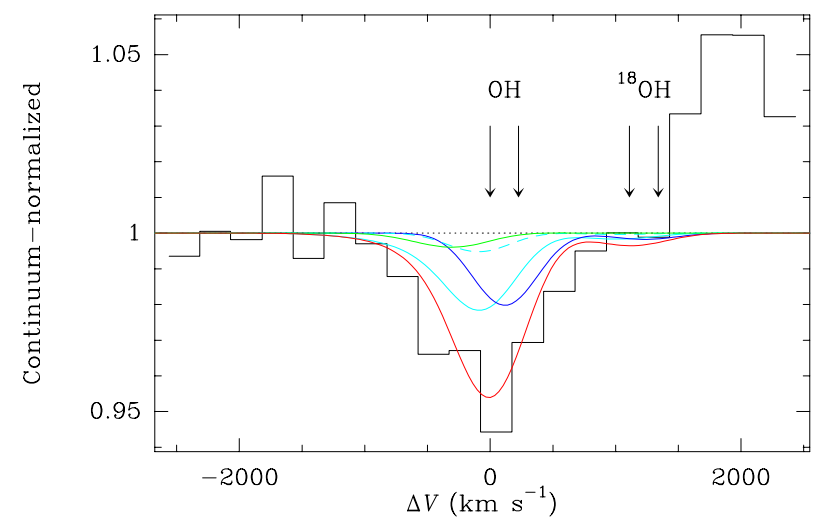

Fig. 13. Model results for the OH35 doublet compared with the observed Spitzer/IRS spectrum. The model and color code are the same as in Fig. 10.

symmetry) and a covering factor of $f=0.20$ (as discussed in Sect. 2.4). The latter value is significantly lower than the covering factor of the compact HVC $(f \gtrsim 0.45)$, possibly indicating that the molecular outflow breaks into clumps as the gas expands from the circumnuclear region. Indeed, the expected average density of $n_{\mathrm{H}} \sim 30 \mathrm{~cm}^{-3}$ is too low to excite the CO 1-0 transition. The fit is reasonable except at central velocities in $\mathrm{OH} 119$, suggesting further scattering in the doublet (see Sect. 3.2). The LEC carries most of the outflowing mass, $M_{\text {gas }} \sim 2 \times 10^{8} \times\left(2.5 \times 10^{-6} / X_{\mathrm{OH}}\right) M_{\odot}$, and most of the mechanical energy, $T_{\text {mech }} \sim 6 \times 10^{56}$ erg (compared with values of the HVC in Table 4). Equation (A.1) gives $\dot{M} \sim 360 \times(2.5 \times$ $\left.10^{-6} / X_{\mathrm{OH}}\right) M_{\odot} \mathrm{yr}^{-1}$ for the above parameters. Within the uncertainties in the analysis of $\mathrm{OH}$ and $\mathrm{CO}$ (Feruglio et al. 2010; Cicone et al. 2012), the energetics inferred from both species appear to be consistent, especially if the $\mathrm{OH}$ abundance drops below our adopted value at large distances from the circumnuclear region.

\subsection{OH35 doublet}

In Fig. 13, the same model used to fit the far-IR OH lines observed with Herschel/PACS (Fig. 10) is compared with the Spitzer/IRS OH35 spectrum. While the absorption at central velocities is reproduced, the model appears to underpredict the absorption at $\sim-500 \mathrm{~km} \mathrm{~s}^{-1}$ as well as at redshifted velocities. Since the ground-state OH53.3 is reproduced, these discrepancies are most likely consequences of the uncertainties in the modeled continuum-flux density at $35 \mu \mathrm{m}$ relative to the flux density at longer wavelengths, which is determined by the solid angle and $T_{\text {dust }}$ of the underlying continuum source. Specifically, we may expect a range of $T_{\text {dust }}$ behind the observed absorption, with the warmest and most compact components contributing significantly to the mid-IR continuum emission.

\section{6. ${ }^{18} \mathrm{OH}$}

One intringuing finding in the $\mathrm{OH}$ spectra of Mrk 231 is the relatively strong absorption by ${ }^{18} \mathrm{OH}$ seen at 120,85 , and $66 \mu \mathrm{m}$. While the ${ }^{18} \mathrm{OH} 120$ doublet may be contaminated by $\mathrm{CH}^{+}$in its blue component and the ${ }^{18} \mathrm{OH} 66$ feature has a probable contribution by $\mathrm{NH}_{2}$ and $\mathrm{H}_{2} \mathrm{O}^{+}$, the prominent ${ }^{18} \mathrm{OH} 85$ is expected to be free from contamination and shows evidence for absorption by outflowing gas as well as by the QC component.

In our model for the $\mathrm{HVC}$ with $T_{\text {dust }}=105 \mathrm{~K}$, we required $N_{18} \mathrm{OH} \sim 1 \times 10^{16} \mathrm{~cm}^{-2}$ to generate the modeled blueshifted absorption in the ${ }^{18} \mathrm{OH} 85$ doublet (Fig. 10c).
Likewise, $N_{18} \mathrm{OH} \sim 1.5 \times 10^{16} \mathrm{~cm}^{-2}$ was obtained for $T_{\text {dust }}=$ $90 \mathrm{~K}$, corresponding to $\mathrm{OH} /{ }^{18} \mathrm{OH} \sim 20$. Similarly, we required for the QC $N_{18} \mathrm{OH} \approx 3 \times 10^{16} \mathrm{~cm}^{-2}$ per unit of $\tau_{50}$, that is, $\mathrm{OH} /{ }^{18} \mathrm{OH} \sim 30$. The overabundance estimated for ${ }^{18} \mathrm{OH}$ is then even more extreme than we previously reported (F10). Since we cannot exclude higher $\mathrm{OH}$ columns at moderate velocities in the outflow (because of saturation in OH84, Fig. A.1c), we favor $\mathrm{OH} /{ }^{18} \mathrm{OH} \sim 20-30$ in both components, with some indications that the ratio decreases in the HVC. Models for the undetected ${ }^{17} \mathrm{OH}$ were also performed, from which we estimate ${ }^{18} \mathrm{OH} /{ }^{17} \mathrm{OH} \gtrsim 5$.

\subsection{More details of the model fit and discrepancies}

There are several spectral features that our modeling does not account for. The high-velocity redshifted emission wing in $\mathrm{OH} 79$ is poorly reproduced, and the redshifted emission feature in OH71 is ignored. The latter may be associated with outflowing gas more excited than modeled for the HVC.

The OH163 is one of the most puzzling of the line shapes (Fig. 10h). The strength of the emission and absorption in this doublet is very sensitive to the continuum opacity. The absorption at blueshifted velocities and the asymmetry between the two lambda-doubling components indicate high continuum opacity and thus suggest a significant contribution by the HVC. However, the narrow linewidths of the emission features would suggest an origin in low-velocity gas, but both the QC and the LEC predict line shapes broader than observed. The dip of emission in between the two lambda-doubling components cannot be reproduced. Since the OH163 doublet is pumped through absorption of far-IR photons, part of the emission is most likely arising from the same region that generates the submillimeter $\mathrm{H}_{2} \mathrm{O}$ emission (G-A10), which is expected to surround the QC (see Sect. 3.2).

\section{Discussion and conclusions}

\subsection{Summary}

The picture that emerges from the $\mathrm{OH}$ observations and models can be summarized as follows: a highly excited component where $\mathrm{OH}$ peaks at central velocities, the $\mathrm{QC}$, represents an outflow-free circumnuclear component with $T_{\text {dust }} \sim 110 \mathrm{~K}$, an effective radius $R \sim 65 \mathrm{pc}$, and a column of $N_{\mathrm{H}} \sim 2 \times 10^{24} \mathrm{~cm}^{-2}$. The observed high-velocity absorption by excited $\mathrm{OH}$ arises from a somewhat larger $\left(R_{\text {int }} \sim 75 \mathrm{pc}, R_{\text {out }} \sim 100 \mathrm{pc}\right.$, both effective radii) radiatively excited and apparently collimated component (the HVC). This component is also associated with high far-IR radiation density $\left(T_{\text {dust }} \sim 100 \mathrm{~K}\right)$ and, given its somewhat larger size, most likely surrounds the QC. This scenario suggests that the QC is feeding the outflow, in the sense that the outflowing gas emanates from the same circumnuclear structure that is responsible for the central-velocity absorption. The $\mathrm{OH}$ column density in the HVC is $N_{\mathrm{OH}} \approx(1.5-3) \times 10^{17} \mathrm{~cm}^{-2}$, suggesting $A_{v} \sim 30$ magnitudes of outflowing circumnuclear gas. We estimate a mass-outflow rate per unit of solid angle in the direction of the observer of at least $\sim 70$ and possibly $\sim 100 M_{\odot} \mathrm{yr}^{-1} \mathrm{sr}^{-1}$ for $X_{\mathrm{OH}}=2.5 \times 10^{-6}$. In spherical symmetry, this would correspond to $\sim 1000 M_{\odot} \mathrm{yr}^{-1}$, though significant departures from a fully spherical model probably reduce the above value by a factor $\sim 2$. The momentum flux attains $\sim 15 L_{\mathrm{AGN}} / c$. In our models, consisting of concentric shells of gas and dust with well-ordered radial motions, the high excitation found for the highest velocity gas was reproduced with a decelerating flow (see discussion in Sect. 3.3.3). An extraordinary enhancement of ${ }^{18} \mathrm{OH}$ was found $\left(\mathrm{OH} /{ }^{18} \mathrm{OH} \lesssim 30\right)$ in both the QC and the HVC. 
Our model for the excited $\mathrm{OH}$ leaves residuals in the groundstate $\mathrm{OH} 119$ and $\mathrm{OH} 79$ doublets, indicating the presence of a low-excitation component of the outflow (the LEC), with a column of $N_{\mathrm{OH}} \approx 7 \times 10^{16} \mathrm{~cm}^{-2}$. The LEC contribution to the profiles (Fig. 12a) is only tentative at low redshifted velocities, but appears to show similar strengths and velocity extents for the absorption and emission features in OH119. This suggests that the LEC is roughly spherical and spatially extended, in contrast with the HVC. If the LEC is extended and surrounds the whole source of $119 \mu \mathrm{m}$ continuum emission, its covering factor is $f \sim 20 \%$, significantly lower than that of the compact HVC ( $f \gtrsim 45 \%$ ), possibly indicating that the molecular outflowing gas breaks into clumps as it moves away from the circumnuclear region. If some of the $\mathrm{OH} 119$ emission is circumnuclear and/or collisionally excited, the covering factor of the extended component must be even lower than $\sim 20 \%$.

\subsection{Torus or thick disk}

The QC has a modeled size (diameter of $\sim 130 \mathrm{pc}$ ) remarkably similar to that of the circumnuclear rotating structure (torus or thick disk) observed with the EVN in $\mathrm{OH}$ megamaser emission by Klöckner et al. (2003), which delineates the central region of the $\mathrm{OH}$ megamaser complex (Richards et al. 2005) and traces the inner region of the radio/H I disk (Carilli et al. 1998) and of the star-forming region observed in the near-IR (Davies et al. 2004, 2007). From the (roughly) estimated continuum optical depth $\left(\tau_{50} \sim 5.5\right)$ and size, the gas mass of the $\mathrm{QC}$ is $\sim 3 \times 10^{8} \times\left(0.01 / X_{\text {dust }}\right) M_{\odot}\left(X_{\text {dust }}\right.$ is the dust-to-gas mass ratio $)^{11}$, significantly higher than the previously estimated virial mass (Klöckner et al. 2003), but still roughly consistent within the uncertainties of both estimates. Furthermore, our inferred $T_{\text {dust }} \sim 110 \mathrm{~K}$ is not far from the value calculated via $\left(L_{\mathrm{AGN}} / 4 \pi R^{2} \sigma\right)^{1 / 4} \sim 130 \mathrm{~K}$, where $L_{\mathrm{AGN}} \sim 2 \times 10^{12} L_{\odot}$ (the actual $T_{\text {dust }}$ will be lower due to opacity effects). We thus tentatively identify the QC component with the circumnuclear OHmegamaser torus (thick disk or oblate spheroid geometries are equally favorable). The match in sizes also suggests that $f$, the covering factor (Table 2), is of order unity for the QC, though more likely $f \sim 0.4-1.0$ for both the QC and HVC because the corresponding absorptions are surely produced in different areas of the warm far-IR surface.

The lack of OH119 absorption at central velocities may be indicative of high densities in the quiescent component, but could also reflect scattering (i.e. reemission in the line) taking place in a flattened structure seen nearly face-on or with low inclination (Sect. 2.5). The geometric problem is probably complex, because high resolution observations indicate a tilt of the torus (Klöckner et al. 2003; Davies et al. 2004; Richards et al. 2005) relative to the outer nearly face-on disk (Downes \& Solomon 1998); nevertheless, the scattering process may be operating in the region responsible for the $\mathrm{H}_{2} \mathrm{O}$ submillimeter emission (which is more extended than the QC, G-A10) or even on relatively large $(\sim 1 \mathrm{kpc})$ scales. Radiative-transfer models in 2D (axial symmetry) are required to distinguish between these scenarios.

\subsection{Radiatively excited molecular outflow}

The HVC is likely to be emanating from, or is at least associated with, this torus, because the highly excited outflowing $a b$ sorbing $\mathrm{OH}$ is seen in front of, and is excited by a strong far-IR

\footnotetext{
11 This gives a gas mass surface density of $2 \times 10^{4} M_{\odot} \mathrm{pc}^{-2}$, which is a lower limit to the total value including stars (Davies et al. 2004, 2007).
}

radiation field most likely generated in and around that circumnuclear component. The QC could also provide a reservoir of gas rich in $\mathrm{OH}$ that feeds the outflow, but if so, then either a relatively smooth acceleration process (e.g. successive low-velocity, non-dissociative $\mathrm{C}$-shocks) allows the $\mathrm{OH}$ to survive, or if the $\mathrm{OH}$ is destroyed in fast (J-) shocks, it must reform in the postshock gas ${ }^{12}$. There is evidence for interaction between the radio jet and the surrounding gas (Ulvestad et al. 1999; Klöckner et al. 2003; Rupke \& Veilleux 2011), as well as an overall (moderate) velocity blueshift of the torus or thick inner disk relative to the surrounding gas at larger spatial scales (Klöckner et al. 2003) that could indicate a slow expansion of the torus. According to outflow models driven by radiation pressure (Roth et al. 2012), the low outward velocity of the gas in the torus can be a result of high inertia, the drop of the radiation pressure with decreasing $T_{\text {dust }}$, and gravitation.

The geometry of the inner outflowing gas (HVC) relative to the torus may be more complex than simulated in our schematic spherically symmetric models. The possibility that the molecular gas is primarily flowing along the polar regions of the torus has two drawbacks; first, the tilt of the torus implies that its axis deviates from the direction of the observer, with the consequent projection effects on the line-of-sight velocity of the polar gas. Second, the gas column along the polar direction is expected to be relatively low, while our inferred high mass-outflow rate and the requirement of absorption of and excitation by optically thick $84 / 65 \mu \mathrm{m}$ continuum indicate large gas reservoirs behind (and associated with) the outflowing gas. The 3D radiation pressure models by Roth et al. (2012) predict the highest differential mass-outflow rates for polar angles $>45^{\circ}$ (their Figs. 12-14), that is, not far from the equatorial plane, and it is just the tilt of the torus that in this context would provide a geometry favorable for detecting high differential mass-outflow rates in the direction of the observer. Conceivably, the observed $\mathrm{OH}$ outflow could probe an interclump medium of the torus itself that is flowing past the dense clumps (possibly probed by the QC), permeating the whole structure. Interaction with the high-density clumps and shadowing effects (Roth et al. 2012) would decelerate the outflowing gas with increasing radial distance. The highest-velocity gas could also be tracing a conical transition region between the torus and the polar directions. In our model for the HVC, the densities for velocities of 500-1500 $\mathrm{km} \mathrm{s}^{-1}$ are $n_{\mathrm{H}} \sim 1000-500 \mathrm{~cm}^{-3}$, respectively, also in rough agreement with the wind-driven outflow models by Faucher-Giguère \& Quataert (2012).

\subsection{Role of the AGN}

The high mass-outflow rate and outflow velocities derived from the far-IR observations of $\mathrm{OH}$ strongly point toward a key role of the central AGN, as previously argued (S11). The momentum flux of $\dot{P} \sim 15 L_{\mathrm{AGN}} / c$ is roughly consistent with that required to regulate the growth of the black hole and set the $M_{\mathrm{BH}}-\sigma$ relation (Debuhr et al. 2012). In the framework of radiation pressure, 3D models indicate that in a clumpy disk with a wide opening angle, the radiation tends to escape along the poles and radiation pressure becomes less efficient, generally accounting for a momentum deposition rate of (1-5) $L_{\mathrm{AGN}} / c$ (Roth et al. 2012). Still, these models predict high differential mass-outflow rates ( $\mathrm{d} \dot{M} / \mathrm{d} \Omega>30 M_{\odot} \mathrm{yr}^{-1} \mathrm{sr}^{-1}$ ) for sufficiently high columns and

12 The transition from a C- to a J-type shock occurs at a critical velocity of $2.76 \mathrm{~B} / \sqrt{4 \pi \rho_{n}}$ (e.g. Ciolek et al. 2004), yielding $<100 \mathrm{~km} \mathrm{~s}^{-1}$ for $B \sim 300 \mu \mathrm{G}$ (Carilli et al. 1998) and a density of $10^{3} \mathrm{~cm}^{-3}$, much lower than the $\mathrm{OH}$ velocities. 
in directions close to the equatorial plane; a high scale-height of the torus/disk, or a relatively high mass-concentration in the polar region, could additionally increase the mass-outflow rate. In addition, fast energy-conserving $\mathrm{AGN}$ winds can do work on the swept-up (molecular) gas and then strongly boost the momentum flux (Faucher-Giguère \& Quataert 2012). It is possible that while radiation pressure affects the whole circumnuclear structure, winds are responsible for the highest velocity wings seen in $\mathrm{OH}$. On the other hand, the high rate of mass loss derived here, together with the narrow-shell configuration favored for the HVC component, may suggest an intermittent (explosive) instead of a steady flow, consistent with the multiple, expanding, concentric supershells seen in the optical/UV at larger scales (Lípari et al. 2005, 2009).

\section{5. ${ }^{18} \mathrm{OH}$ and the circumnuclear star formation}

An intriguing implication of the present observations is the strong enhancement of ${ }^{18} \mathrm{OH}$ in both the QC and the HVC. Since fractionation effects do not chemically enhance ${ }^{18} \mathrm{OH}$ (Langer et al. 1984), the ${ }^{16} \mathrm{OH} /{ }^{18} \mathrm{OH}$ ratio is expected to be the same as the ${ }^{16} \mathrm{O} /{ }^{18} \mathrm{O}$ ratio. Our results indicate that ${ }^{18} \mathrm{O}$ is enhanced by about one order of magnitude relative to the Galactic Sgr B2 (Polehampton et al. 2005), and even more relative to the solar value. This is of interest in the context of the high metallicities that are observed in quasar environments, whose enrichment is thought to be due to star formation with an IMF weighted toward massive stars (see review by Hamann et al. 2007). Similarly, ${ }^{18} \mathrm{O}$ is thought to be enriched in the ISM by partial He burning in massive stars (e.g. Wilson \& Matteucci 1992; Henkel \& Mauersberger 1993; Prantzos et al. 1996; Wouterloot et al. 2008; Kobayashi et al. 2011), and the stars in the inner disk of Mrk 231 have been formed in situ (Davies et al. 2004). This is consistent with the lack of detection of ${ }^{17} \mathrm{OH}$ if ${ }^{17} \mathrm{O}$ is primarily produced in low- and intermediate-mass stars (Sage et al. 1991; Wilson \& Matteucci 1992) that, regardless of the IMF, are not expected to have a significant chemical effect on the ISM of Mrk 231 (Muller et al. 2006) given the youth of the circumnuclear starburst, $\lesssim 0.25$ Gyr (Davies et al. 2007). Artymowicz et al. (1993) and Collin \& Zahn (1999) have proposed and explored a scenario in which unstable fragments trapped in the accretion disk of massive black holes, grow by accretion to $\sim 50-100 M_{\odot}$ stars and ultimately explode as supernovae ( $\mathrm{SNe}$ ), to explain the metal enrichment of QSO ejecta, though it is unclear whether the ${ }^{18} \mathrm{O}$ would be destroyed in these conditions by He burning, yielding ${ }^{22} \mathrm{Ne}$ (e.g. Prantzos et al. 1996). Interestingly, a very low (but not so extreme) ${ }^{16} \mathrm{O} /{ }^{18} \mathrm{O} \sim 50$ ratio, together with a high ${ }^{18} \mathrm{O} /{ }^{17} \mathrm{O} \sim 12$ ratio, are also inferred in the arm of a spiral galaxy at $z=0.89$ (Muller et al. 2006, 2011). In Mrk 231, ${ }^{18} \mathrm{OH}$ is detected up to a velocity shift of $\sim-600 \mathrm{~km} \mathrm{~s}^{-1}$, although ${ }^{18} \mathrm{OH}$ enhancement at higher velocities is not ruled out. While the high column density of ${ }^{18} \mathrm{OH}$ in the QC indicates previous ${ }^{18} \mathrm{O}$ enrichment of the swept-up gas ${ }^{13}$, the possible relative enhancement of ${ }^{18} \mathrm{OH}$ that we inferred in the line wing could suggest the contribution of $\mathrm{SNe}$ or massive stellar winds to the outflow. More

\footnotetext{
${ }^{13}$ Scaling the results by Davies et al. (2007) for an estimated SN rate of $\sim 4 \mathrm{yr}^{-1}$ within the inner $\sim 500 \mathrm{pc}$ (Davies et al. 2004), the cumulative ejected mass is $\sim 2 \times 10^{9} M_{\odot}$ (including OB winds and AGB stars, Davies et al. 2007), which is similar to the current stellar mass (Davies et al. $2004)$ and also similar to the total gas mass $\left(\sim 1.8 \times 10^{9} M_{\odot}\right.$, Downes $\&$ Solomon 1998). If a significant fraction of these ejecta still remains bound, the circumnuclear ISM is expected from these grounds to be deeply recycled by the ejecta of high-mass stars.
}

studies of $\mathrm{OH} /{ }^{18} \mathrm{OH}$ in galaxies and $\mathrm{SNe}$ are required to fully understand the evolutionary implications of these enhancements.

Acknowledgements. PACS has been developed by a consortium of institutes led by MPE (Germany) and including UVIE (Austria); KU Leuven, CSL, IMEC (Belgium); CEA, LAM (France); MPIA (Germany); INAFIFSI/OAA/OAP/OAT, LENS, SISSA (Italy); IAC (Spain). This development has been supported by the funding agencies BMVIT (Austria), ESA-PRODEX (Belgium), CEA/CNES (France), DLR (Germany), ASI/INAF (Italy), and CICYT/MCYT (Spain). E.G.-A. is a Research Associate at the Harvard-Smithsonian Center for Astrophysics, and thanks the Spanish Ministerio de Economía y Competitividad for support under projects AYA2010-21697-C05-0 and FIS2012-39162-C06-01. Basic research in IR astronomy at NRL is funded by the US ONR; J.F. and H.W.W.S. also acknowledge support from the NHSC. S.V. thanks NASA for partial support of this research via Research Support Agreement RSA 1427277, support from a Senior NPP Award from NASA, and his host institution, the Goddard Space Flight Center, and acknowledges support from the Alexander von Humboldt Foundation for a renewed visit to Germany following the original 2009 award. This research has made use of NASA's Astrophysics Data System (ADS) and of GILDAS software (http: //www . iram. fr/IRAMFR/GILDAS).

\section{Appendix A: Some properties of the $\mathrm{OH}$ outflow models}

In our models, which assume a constant mass-outflow rate and velocity gradient within $R_{\text {int }}$ and $R_{\text {out }}$, the relationship between the mass-outflow rate per unit of solid angle $(\mathrm{d} \dot{M} / \mathrm{d} \Omega)$ and $N_{\mathrm{OH}}$ is given by (from Eqs. (2) and (3))

$$
\begin{aligned}
\mathrm{d} \dot{M} / \mathrm{d} \Omega= & f m_{\mathrm{H}} X_{\mathrm{OH}}^{-1} v_{\text {int }} R_{\text {int }} N_{\mathrm{OH}} \times\left(\frac{x-v_{\text {out }} / v_{\text {int }}}{x-1}\right) \\
& \times\left[1-\frac{1}{x}+\frac{v_{\text {int }} / v_{\text {out }}-1}{v_{\text {int }} x / v_{\text {out }}-1} \ln \left(\frac{v_{\text {int }} x}{v_{\text {out }}}\right)\right]^{-1},
\end{aligned}
$$

where $x \equiv R_{\text {out }} / R_{\text {int }}$. For given $N_{\mathrm{OH}}, R_{\text {int }}, v_{\text {int }}$, and $v_{\text {out }}$, both $\dot{M}$ and the $\mathrm{OH}$ excitation increase with decreasing $x$ (Fig. 11a), so that higher excitation (e.g. higher OH65/OH84 ratio) implies a more compact outflow and an increasing $\dot{M}$ in our models.

The $\mathrm{OH}$ column density per unit of velocity interval is

$$
\frac{\mathrm{d} N_{\mathrm{OH}}}{\mathrm{d} v}=\frac{\dot{M} X_{\mathrm{OH}} R_{\mathrm{int}}}{4 \pi g\left(p_{\mathrm{f}}\right) f m_{\mathrm{H}}} \times \frac{x-1}{\left|v_{\text {out }}-v_{\text {int }}\right|} \times \frac{1}{r^{2} v(r)} .
$$

The corresponding $N_{\mathrm{H}}$ spectrum, calculated in velocity intervals of $100 \mathrm{~km} \mathrm{~s}^{-1}$, is shown in Fig. A.1a for the model of the HVC displayed in Fig. 10a. In a compact decelerating outflow, the column density remains nearly constant for high velocities. The visual extinction at high velocities in these $100 \mathrm{~km} \mathrm{~s}^{-1}$ intervals is expected to be $A_{V} \sim 1 \mathrm{mag}$.

The modeled line shapes depend on the velocity distribution of $N_{\mathrm{OH}}$ and of the $\mathrm{OH}$ excitation, and on the covering factor as a function of the line-of-sight velocity. The increasing excitation with increasing velocity shifts is obtained in our models with a decelerating field (Fig. A.1b). The calculated OH84 and OH65 optical depths along a radial path are shown in Fig. A.1c, indicating saturation effects in the $\mathrm{OH} 84$ doublet mostly at moderate velocities, but optically thin absorption in OH65. On the other hand, the steep decrease of the OH84 absorption with increasing velocity shift (Fig. 10) is indicative of a decreasing covering factor with increasing projected velocity, as shown in Fig. A.1d. At low projected velocities $\left(<400 \mathrm{~km} \mathrm{~s}^{-1}\right)$, the covering factor exceeds unity, which generates reemission from the limb of the outflow at significantly redshifted velocities. Since this reemission is not observed in $\mathrm{OH} 84$, a collimated $\left(p_{\mathrm{f}} \sim R_{\text {int }}\right)$ outflow is favored. 
E. González-Alfonso et al.: The Mrk 231 molecular outflow as seen in $\mathrm{OH}$

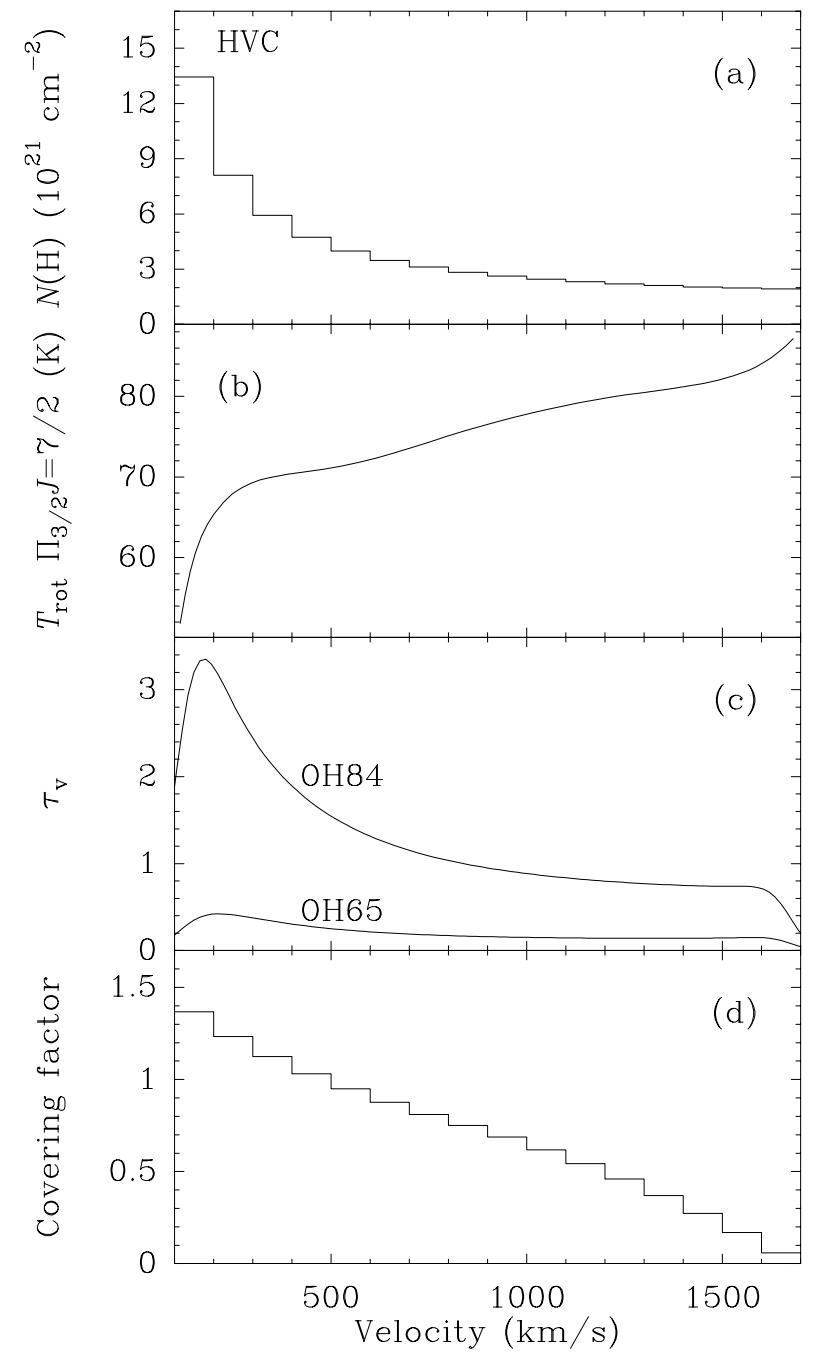

Fig. A.1. Details of the model for the HVC shown in Fig. 10 with light-blue curves; note that higher velocities correspond to lower distances to the far-IR exciting source in a decelerating field. a) Column density of $\mathrm{H}$ nuclei (assuming $X_{\mathrm{OH}}=2.5 \times 10^{-6}$ ) in intervals of $100 \mathrm{~km} \mathrm{~s}^{-1}$ as a function of the radial velocity for $R_{\text {out }} / R_{\text {int }}=1.3$, $N_{\mathrm{OH}}=1.6 \times 10^{17} \mathrm{~cm}^{-2}, v_{\text {int }}=1700 \mathrm{~km} \mathrm{~s}^{-1}$, and $v_{\text {out }}=100 \mathrm{~km} \mathrm{~s}^{-1}$. b) Rotational temperature of the $\Pi_{3 / 2} J=7 / 2$ level (the lower level of the OH65 transition) relative to the ground state, as a function of the radial velocity. c) $\mathrm{OH} 84$ and $\mathrm{OH} 65$ optical depths along a ray passing through the center, and d) covering factor of the continuum, both as a function of the line-of-sight velocity. At low projected velocities $\left(<400 \mathrm{~km} \mathrm{~s}^{-1}\right)$, the covering factor exceeds unity, which generates reemission from the limb of the outflow.

The total mass-outflow rate is given by

$$
\dot{M}=4 \pi g\left(p_{\mathrm{f}}\right) \mathrm{d} \dot{M} / \mathrm{d} \Omega,
$$

where $g=1$ for $p_{\mathrm{f}}=R_{\text {out }}$. For $R_{\text {int }} \leq p_{\mathrm{f}}<R_{\text {out }}$, we roughly approximate the geometry depicted in Fig. 8 as two cones, each one with half opening angle $\sin \theta_{1 / 2}=p_{\mathrm{f}} / R_{\text {out }}$, and thus

$g\left(p_{\mathrm{f}}\right)=1-\sqrt{1-\left(p_{\mathrm{f}} / R_{\mathrm{out}}\right)^{2}}$.

This approximation underestimates $\dot{M}$ because the model still includes the contribution by gas outflowing along the plane of sky (Fig. 8).

\section{References}

Aalto, S., García-Burillo, S., Muller, S., et al. 2012, A\&A, 537, A44

Adams, F. C. 1991, ApJ, 382, 544

Amari, S., Zinner, E., \& Lewis, R. S. 1995, ApJ, 447, L147

Armus, L., Charmandaris, V., \& Bernard-Salas, J. 2007, ApJ, 656, 148 Artymowicz, P., Lin, D. N. C., \& Wampler, E. J. 1993, ApJ, 409, 592 Baan, W. A., Haschick, A. D., \& Henkel, C. 1989, ApJ, 346, 680

Carilli, C. L., Wrobel, J. M., \& Ulvestad, J. S. 1998, AJ, 115, 928

Chevalier, R. A. 1977, ARA\&A, 15, 175

Cicone, C., Feruglio, C., Maiolino, R., et al. 2012, A\&A, 543, A99

Ciolek, G. E., Roberge, W. G., \& Mouschovias, T. C. 2004, ApJ, 610, 781

Collin, S., \& Zahn, J.-P. 1999, A\&A, 344, 433

Davies, R. I., Tacconi, L. J., \& Genzel, R. 2004, ApJ, 613, 781

Davies, R. I., Müller Sánchez, F., Genzel, R., et al. 2007, ApJ, 671, 1388

Debuhr, J., Quataert, E., \& Ma, C.-P. 2012, MNRAS, 420, 2221

Di Matteo, T., Springel, V., \& Hernquist, L. 2005, Nature, 433, 604

Downes, D., \& Solomon, P. M. 1998, ApJ, 507, 615

Farrah, D., Bernard-Salas, J., Spoon, H. W. W., et al. 2007, ApJ, 667, 149

Faucher-Giguère, C.-A., \& Quataert, E. 2012, MNRAS, 425, 605

Feruglio, C., Maiolino, R., Piconcelli, E., et al. 2010, A\&A, 518, L155

Fischer, J., Sturm, E., González-Alfonso, et al. 2010, A\&A, 518, L41 (F10)

González-Alfonso, E., \& Cernicharo, J. 1997, A\&A, 322, 938

González-Alfonso, E., \& Cernicharo, J. 1999, ApJ, 525, 845

González-Alfonso, E., Cernicharo, J., van Dishoeck, E. F., Wright, C. M., \& Heras, A. 1998, ApJ, 502, L169

González-Alfonso, E., Smith, H. A., Ashby, et al. 2008, ApJ, 675, 303

González-Alfonso, E., Fischer, J., Isaak, K., et al. 2010, A\&A, 518, L43

González-Alfonso, E., Fischer, J., Graciá-Carpio, J., et al. 2012, A\&A, 541, A4 (G-A12)

González-Alfonso, E., Fischer, J., Bruderer, S., et al. 2013, A\&A, 550, A25 (GA13)

Hamann, F., Warner, C., Dietrich, M., \& Ferland, G. 2007, ASP Conf. Ser., 373, 653

Henkel, C., \& Mauersberger, R. 1993, A\&A, 274, 730

Klöckner, H.-R., Baan, W. A., \& Garrett, M. A., 2003, Nature, 421, 821

Kobayashi, C., Karakas, A. I., \& Umeda, H. 2011, MNRAS, 414, 3231

Langer, W. D., Graedel, T. E., Frerking, M. A., \& Armentrout, P. B. 1984, ApJ, 277,581

Lípari, S., Terlevich, R., Zheng, W., et al. 2005, MNRAS, 360, 416

Lípari, S., Sánchez, S. F., Bergmann, M., et al. 2009, MNRAS, 392, 1295

Meijerink, R., Spaans, M., Loenen, A. F., \& van der Werf, P. P. 2011, A\&A, 525, A119

Müller, H. S. P., Thorwirth, S., Roth, D. A., \& Winnewisser, G. 2001, A\&A, 370, L49

Müller, H. S. P., Schlöder, F., Stutzki, J., \& Winnewisser, G. 2005, J. Mol. Struct., 742,215

Muller, S., Guélin, M., Dumke, M., Lucas, R., \& Combes, F. 2006, A\&A, 458, 417

Muller, S., Beelen, A., Guélin, M., et al. 2011, A\&A, 535, A103

Murray, N., Quataert, E., \& Thompson, T. A. 2005, ApJ, 618, 569

Offer, A. R., \& van Hemert, M. C. 1992, Chem. Phys., 163, 83

Pickett, H. M., Poynter, R. L., Cohen, E. A., et al. 1998, J. Quant. Spectr. Rad. Transf., 60, 883

Pilbratt, G. L., Riedinger, J. R., Passvogel, T., et al. 2010, A\&A, 518, L1

Poglitsch, A., Waelkens, C., Geis, N., et al. 2010, A\&A, 518, L2

Polehampton, E. T., Brown, J. M., Swinyard, B. M., \& Baluteau, J.-P. 2003, A\&A, 406, L47

Polehampton, E. T., Baluteau, J. -P., \& Swinyard, B. M. 2005, A\&A, 437, 957

Prantzos, N., Aubert, O., \& Audouze, J. 1996, A\&A, 309, 760

Richards, A. M. S., Knapen, J. H., Yates, J. A., et al. 2005, MNRAS, 364, 353

Roth, N., Kasen, D., Hopkins, P. F., \& Quataert, E. 2012, ApJ, 759, 36

Rupke, D. S. N., \& Veilleux, S. 2011, ApJ, 729, L27

Sage, L. J., Henkel, C., \& Mauersberger, R. 1991, A\&A, 249, 31

Sakamoto, K., Aalto, S., Wilner, D. J., et al. 2009, ApJ, 700, L104

Spoon, H. W. W., \& Holt, J. 2009, ApJ, 702, L42

Spoon, H. W. W., Farrah, D., Lebouteiller, V., et al. 2013, ApJ, 775, 127

Sturm, E., González-Alfonso, E., Veilleux, S., et al. 2011, ApJ, 733, L16 (S11)

Ulvestad, J. S., Wrobel, J. M., \& Carilli, C. L. 1999, ApJ, 516, 127

van der Werf, P. P., Isaak, K. G., Meijerink, R., et al. 2010, A\&A, 518, L42

Veilleux, S., Cecil, G., \& Bland-Hawthorn, J. 2005, ARA\&A, 43, 769

Veilleux, S., Rupke, D. S. N., Kim, D.-C., et al. 2009, ApJS, 182, 628

Veilleux, S., Meléndez, M., Sturm, E., et al. 2013, ApJ, 776, 27

Walter, F., Weiss, A., \& Scoville, N. 2002, ApJ, 580, L21

Wilson, T. L., \& Matteucci, F. 1992, A\&AR, 4, 1

Wouterloot, J. G. A., Henkel, C., Brand, J., \& Davis, G. R. 2008, A\&A, 487, 237 